\title{
On the determination of fully conjugate hypoid tooth flanks
}

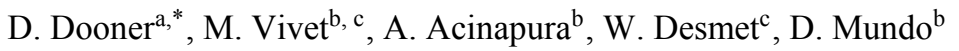 \\ ${ }^{a}$ Department of Mechanical Engineering, University of Puerto Rico at Mayagüez, Mayagüez, Puerto Rico 00681 \\ ${ }^{\mathrm{b}}$ Department of Mechanical, Energy, and Management Engineering, University of Calabria, Ponte P. Bucci, 87036 Rende (Italy) \\ ${ }^{\mathrm{c}}$ Department of Mechanical Engineering, Katholieke Universiteit Leuven, Celestijnenlaan 300B, B-3001 Heverlee (Leuven), Belgium
}

\begin{abstract}
Presented is a procedure to individually specify fully conjugate hypoid gear tooth flanks. Cartesian coordinates for the hypoid tooth flanks are derived in terms of a planar rack with a specified constant pressure angle. These coordinates define a "coordinate curve" on the tooth flank. Each coordinate curve depends on an axial coordinate and the entire tooth flank is established as a family of these coordinate curves. This process results in global interference between locally conjugate hypoid tooth flanks if the pressure angle is constant. A differential equation is established for the axial variation in the rack's pressure angle to avoid global interference. The resulting axial variation in normal pressure angle is combined with the determination of coordinate curves to obtain fully conjugate tooth flanks. An automotive hypoid gear pair is presented to showcase the process. Unloaded Ease-off (UEO) topography is used to quantify conjugate action for the example hypoid gear pair. Subsequently, axial and transverse profile modifications are applied individually to the ideal tooth flanks to accommodate generalized loading conditions. Loaded Transmission Error (TE) and maximum contact pressure are reported for a set of input torques.
\end{abstract}

Keywords: Hypoid gears · Cylindroidal coordinates · Higher order reciprocity · Exact conjugate motion · Transmission error · Profile modification

\section{Introduction}

Deproximating Tredgold's Approximation presented a background on the historical development of Tredgold's Approximation along with a level of current research on the kinematic geometry of spatial gearing [1]. Additional background literature on spatial tooth flanks is reported in [2]. Presented in this article is an extension of Deproximating Tredgold's Approximation used to specify conjugate tooth flanks for bevel gears. To the authors' awareness, no known procedure exists to singly specify fully conjugate tooth surfaces for hypoid gear sets. Such a procedure can help establish geometric limitations for hypoid flanks, define reference surfaces for inspection, and help enhance current gear manufacturing capabilities (e.g., swarf milling, contour milling, 3D printing, molding, cold rolling, forging, powder metallurgy, hobbing as well as face-cutting).

The new content in this manuscript focuses on skew axis gearing with constant speed ratio (viz., hypoid gearing). Presented in [2] was a methodology for establishing first order and second order reciprocity between the tooth flank normal and the ISA defined by the rotation axes and speed ratio. This higher order conjugacy was based on a variation of the pressure angle in the transverse direction while constant in the axial direction. The result was a near-zero $(\approx 0.1 \mu \mathrm{m})$ UEO topography for a banded strip centered along the tooth flank. In this manuscript, fully conjugate hypoid tooth flanks are obtained by allowing the pressure angle to vary in the axial direction while constant along a transverse curve. The result is exact zero UEO topography spanning the entire tooth flank.

\footnotetext{
* Corresponding author at: Department of Mechanical Engineering, University of Puerto Rico - Mayagüez, Mayagüez, Puerto Rico 00681

E-mail address: david.dooner@upr.edu
} 
Table 1. Related quotations on hypoid gear geometry.

\begin{tabular}{|c|c|}
\hline Reference & Quote \\
\hline $\begin{array}{l}{[4]} \\
\text { Page } 170\end{array}$ & $\begin{array}{l}\text { "The theory cannot even be considered as yet settled, for writers upon theoretical mechanism } \\
\text { do not agree upon it, and there are points yet in controversy. }\end{array}$ \\
\hline Page 171 & $\begin{array}{l}\text {... But for the skew bevel gear there is no analogous process, for it is impossible to imagine } \\
\text { a surface of such nature that it can be made to coincide with a similar surface when both are } \\
\text { attached to revolving askew shafts." }\end{array}$ \\
\hline $\begin{array}{l}{[5]} \\
\text { Page } 17\end{array}$ & $\begin{array}{l}\text { "We see that } \kappa \text { and } \tau \text { have the dimension } \mathrm{L}^{-1} \text {. Where }\left|\kappa^{-1}\right|=|\mathrm{R}| \text { is called the radius of } \\
\text { curvature, }\left|\tau^{-1}\right|=|\mathrm{T}| \text { is called the radius of torsion. However, this quantity }|\mathrm{T}| \text { does not admit } \\
\text { of such a ready and elegant geometrical interpretation as }|\mathrm{R}| . "\end{array}$ \\
\hline $\begin{array}{l}{[6]} \\
\text { Page } 89\end{array}$ & $\begin{array}{l}\text { "...; but apart from limited success, nobody has yet succeeded in fully developing a unified } \\
\text { axode-based theory in which curvature and the like are expressible in terms comparable to those } \\
\text { for the plane. Enormous difficulties are encountered, even when Krames (1937-8) starts with } \\
\text { axodes of relatively simple geometrical forms." }\end{array}$ \\
\hline $\begin{array}{l}{[7]} \\
\text { Page } 161\end{array}$ & $\begin{array}{l}\text { "The relationships between the local properties of the axodes (and their striction curves) and } \\
\text { the higher order instantaneous invariants do not seem to have been developed." }\end{array}$ \\
\hline $\begin{array}{l}{[8]} \\
\text { Page } 417\end{array}$ & $\begin{array}{l}\text { "It is interesting to note that, for a crossed helical gear pair, there is no relation between the } \\
\text { pitch cylinder radii and the center distance C." }\end{array}$ \\
\hline [9] & $\begin{array}{l}\text { "I have said in the Prologue ( } § \text { P.05 et seq.) and elsewhere in this book that there is no place in } \\
\text { general spatial involute theory for the concept pitch surface, as though it were somehow the } \\
\text { spatial analogue of the simpler concept pitch circle in the plane; ..." }\end{array}$ \\
\hline $\begin{array}{l}{[10]} \\
\text { Page } 679\end{array}$ & $\begin{array}{l}\text { "The concept of axodes of hypoid gears has found a limited application in design and is used } \\
\text { merely for visualization of relative velocity. The main reason for this is that the location of } \\
\text { axodes is out of the zone of meshing of hypoid gears." }\end{array}$ \\
\hline [11] & $\begin{array}{l}\text { "The modeling of the tooth flanks of gears with skew axes, however, still represents a } \\
\text { challenge to geometers. Hence, there is a need to develop algorithms for the geometric } \\
\text { modeling of these gears." }\end{array}$ \\
\hline
\end{tabular}

It is important to clarify the statement on any known procedure to obtain conjugate hypoid flanks. One related procedure is to define a surface for one of the two gear elements (e.g., the input gear). The Cartesian Coordinates for any point on this surface together with the surface normal of this point can be combined to define a conjugate image point on a mating output gear flank. This process is repeated for a set of points on the input gear flank resulting in a set of points that define a conjugate output flank. This procedure is typically used to establish Ease-off topography. This procedure is simple yet not amenable to establishing reference involute tooth surfaces as usually defined for spur and helical gear sets with constant pressure angle flanks.

Recognizing that gears have been in existence for over 2,000 years with the South Pointing Chariot (circa 200-265 $\mathrm{AD}$ ) and the Antikythera Mechanism (circa 150-100 BC), one might be surprised by the differences between theoretical kinematics and the current design and fabrication of hypoid gears. A face cutter process is typically used to mass produce spiral bevel and hypoid gears [3]. These differences are better appreciated by recognizing that the face cutting process is an iterative based process that optimizes tooth contact. This iterative process is sometimes referred to as gear art. Listed chronologically by publication date in Table 1 are quotes that highlight challenges describing kinematic relations inherent in hypoid gearing. Two key limitations exist in today's design and manufacture of hypoid gears. One limitation expressed by researchers in this area and cited in Table 1 involves an incomplete understanding of hypoid gear kinematics. The other limitation involves the manufacturing process used to fabricate hypoid gears. One goal of this article is to understand the kinematic geometry of spatial gearing and propose a hypoid gear design methodology based on this understanding. Consequently, the focus of this effort is with hypoid gear geometry and not the manufacturing process used to produce hypoid gears. A description of gear teeth begins with an overview of cylindroidal coordinates.

\section{Cylindroidal coordinates}

Motion transmission between parallel axes is the most common form of gearing. Such gear forms are oftentimes analyzed using a plane perpendicular to the parallel rotation axes together with a plane coincident with the parallel rotation axes. A plane perpendicular to the rotation axes is commonly referred to as a transverse plane and the plane coincident with the rotation axes is referred to as the axial plane. No known generalization to transverse and axial surfaces exists for hypoid gears. Cylindroidal coordinates were introduced to describe gear teeth for generalized spatial 

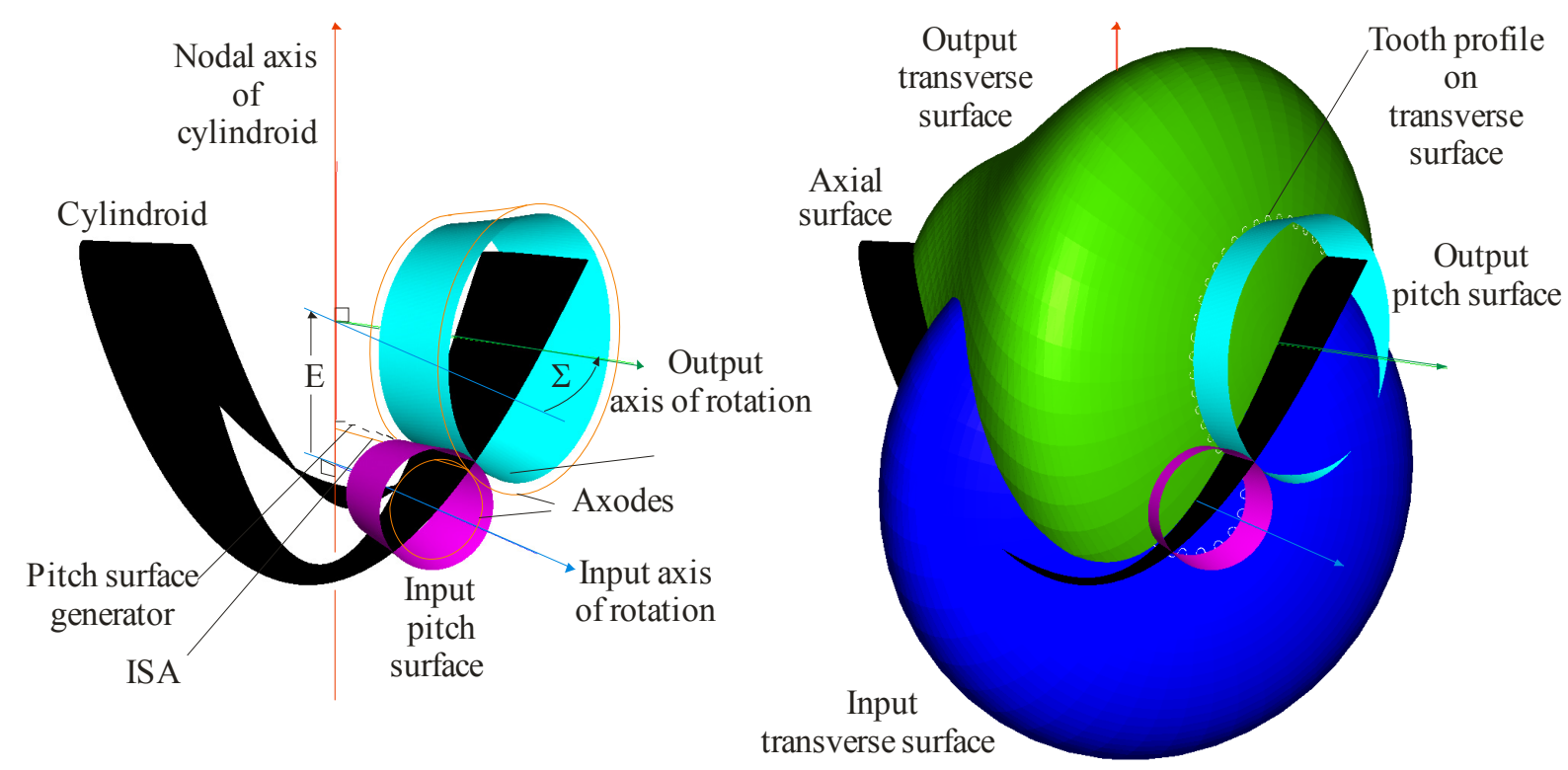

Fig. 1. Systems of cylindroidal coordinates for hypoid gear pair for uniform motion.

gear elements [12]. Cylindroidal coordinates are used to define pitch, transverse and axial surfaces for hypoid gears. The transverse and axial surfaces were established as a corollary to the transverse and axial plane of parallel axis gearing.

Depicted in Fig. 1 are an input axis of rotation, an output axis of rotation, the cylindroid uniquely defined by these two axes together with two pitch surfaces. Overall gear geometry is governed by the axes offset E and axes angle $\Sigma$. One pitch surface is the input pitch surface and the mating pitch surface is the output pitch surface. The two pitch surfaces are tangent along a line; this line is coincident with the cylindroid. In general, this line is not the ISA (Instantaneous Screw Axis) and the axodes are not pitch surfaces. Also shown in Fig. 1 are two systems of curvilinear coordinates defined by the cylindroid; hence the name cylindroidal coordinates. The axial surface is the cylindroid defined by the two gear axes of rotation. Gear geometry will be determined using two (viz, input and output) systems of cylindroidal coordinates. Cylindroidal coordinates can be used to describe any tooth profile; namely, involute, cycloidal, or circular-arc. The gear set is "hypoid" when both E and $\Sigma$ are non-zero. The term "hypoid" is a shortening of "hyp[erbol]oid". We have three gear cases. Each case can be further divided into constant speed and variable speed ratio (viz., non-circular gears). A constant speed ratio is used to illustrate cylindriodal coordinates for the three cases. The three cases are defined by 17 teeth on the input gear element, 41 teeth on the output gear element, 1.6 axial contact ratio, and $10 \mathrm{~mm}$ face width. Each input gear has a left-hand spiral.

Case I involves general skew axis gearing, also referred to as hypoid, spatial, and rear axis gearing. Skew axis gearing is defined by $\Sigma \neq 0^{\circ}$ and $\mathrm{E} \neq 0 \mathrm{~mm}$. To help elucidate the need for a system of coordinates, we will ask, what is the infinitesimally thin surface (transverse surface) to draw teeth for transmitting motion between skew axes via surfaces in direct contact or what is the transverse contact ratio for skew axis gearing? For this generalized scenario, the transverse surfaces are based on the cylindroid. The axial surface is a general cylindroid and coincident with the two skew axes of rotation. The pitch surfaces are always hyperboloidal; namely, single sheet hyperboloids with circular throats. Their sizes depend on the speed ratio together with the instantaneous pressure angle and spiral angle. The infinitely many transverse surfaces determine the face width of the gear. Illustrated in Fig. 2a are two systems of cylindroidal coordinates, defined by $\Sigma=30^{\circ}$ and offset $\mathrm{E}$ equal to twice the face width. Also shown in Fig. $2 \mathrm{a}$ is a sample gear set based on the cylindroidal coordinates. The hyperboloidal pitch surfaces are left-handed. The intersection between the gear element and one of its transverse surfaces is superimposed on the displayed transverse surface. The intersection between a transverse surface and the pitch surface is a circular transverse curve.

Case II involves parallel axis gearing, also referred to as cylindrical and planar gears (i.e., spur and helical). Parallel axis gearing is defined by $\Sigma=0^{\circ}$ and $\mathrm{E} \neq 0 \mathrm{~mm}$. For this special case, the transverse surfaces are flat planes perpendicular to the two parallel axes of rotation. The axial surface is a special cylindroid; it is a planar surface perpendicular to the transverse surfaces and coincident with the two parallel axes of rotation. The pitch surfaces are always cylindrical and coincide with the axodes (centrodes). Their sizes depend on the speed ratio and are independent of the pressure angle and the spiral angle. The infinitely many planar transverse surfaces determine the face width of the gear. Illustrated in Fig. 2b are two systems of cylindrical coordinates, defined by the special case of cylindroidal coordinates where $\Sigma=0^{\circ}$ and offset $\mathrm{E}$ equals twice the face width. Also shown in Fig. 2b is a sample gear set based on the special cylindroidal coordinates. The intersection between each gear element and one of its planar transverse surfaces is superimposed on the displayed planar transverse surface. 

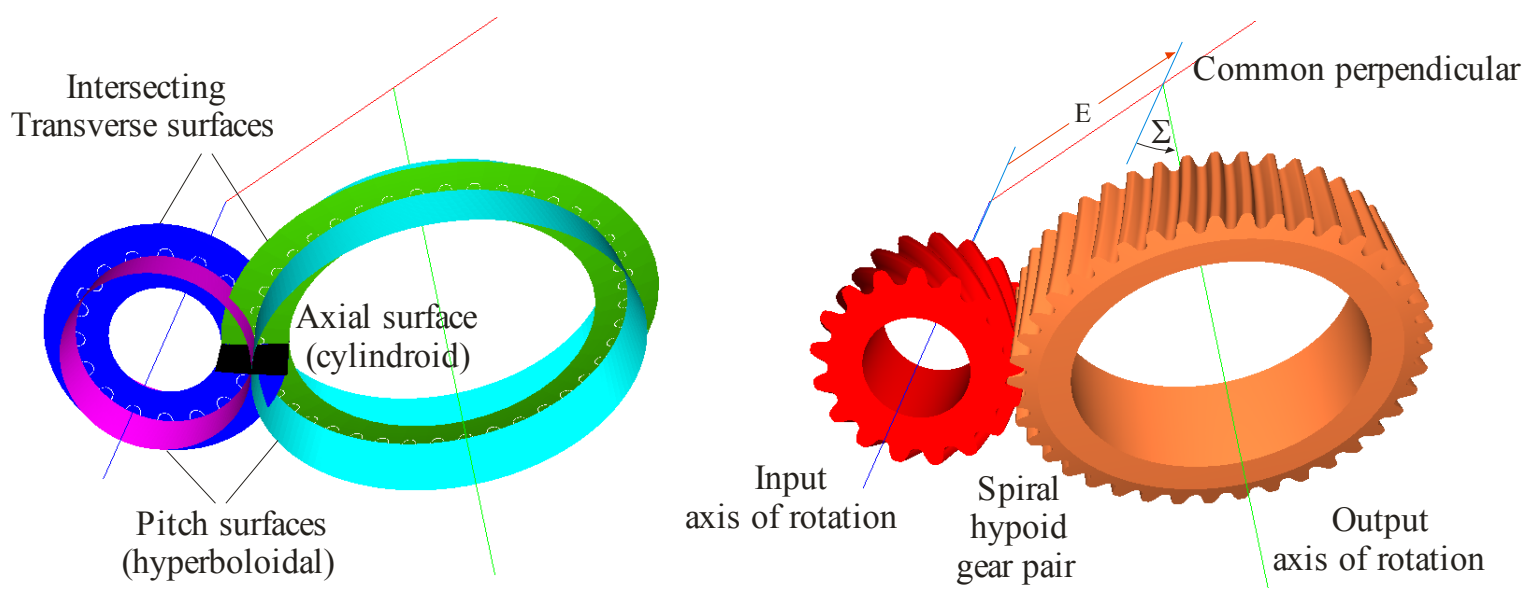

(a) Skew axes for input and output gears.
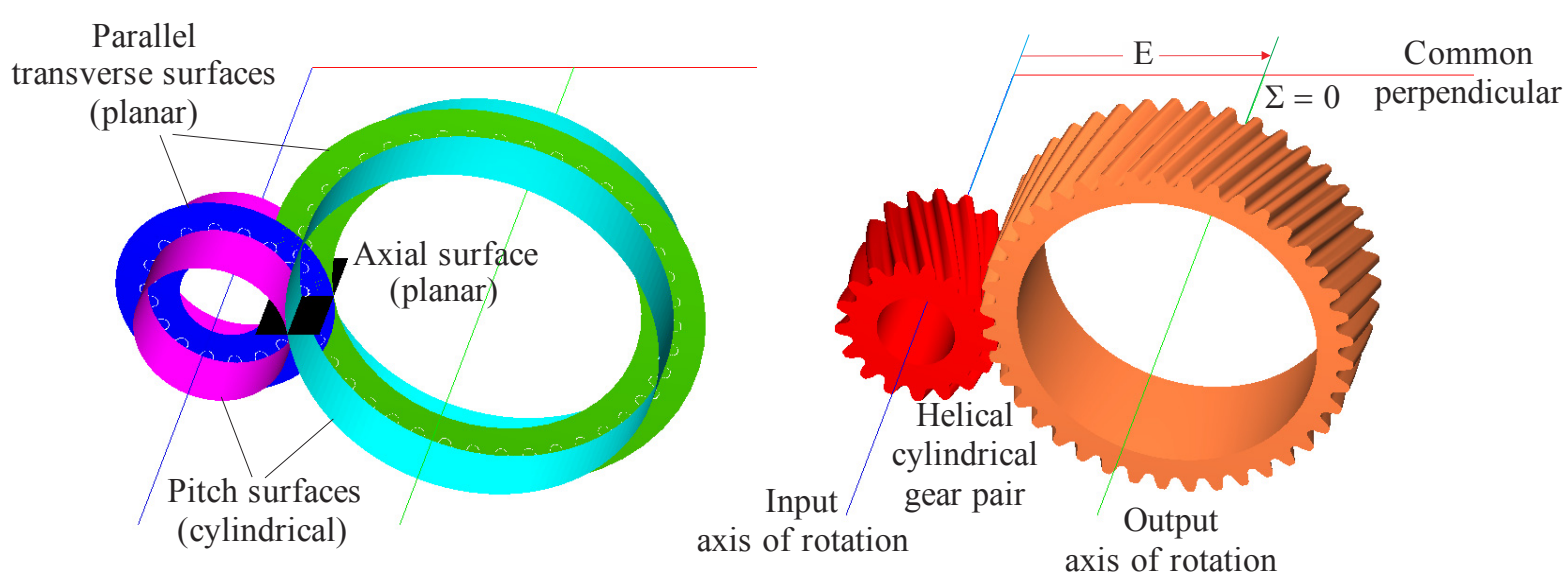

(b) Parallel axes for input and output gears (cylindrical coordinates).
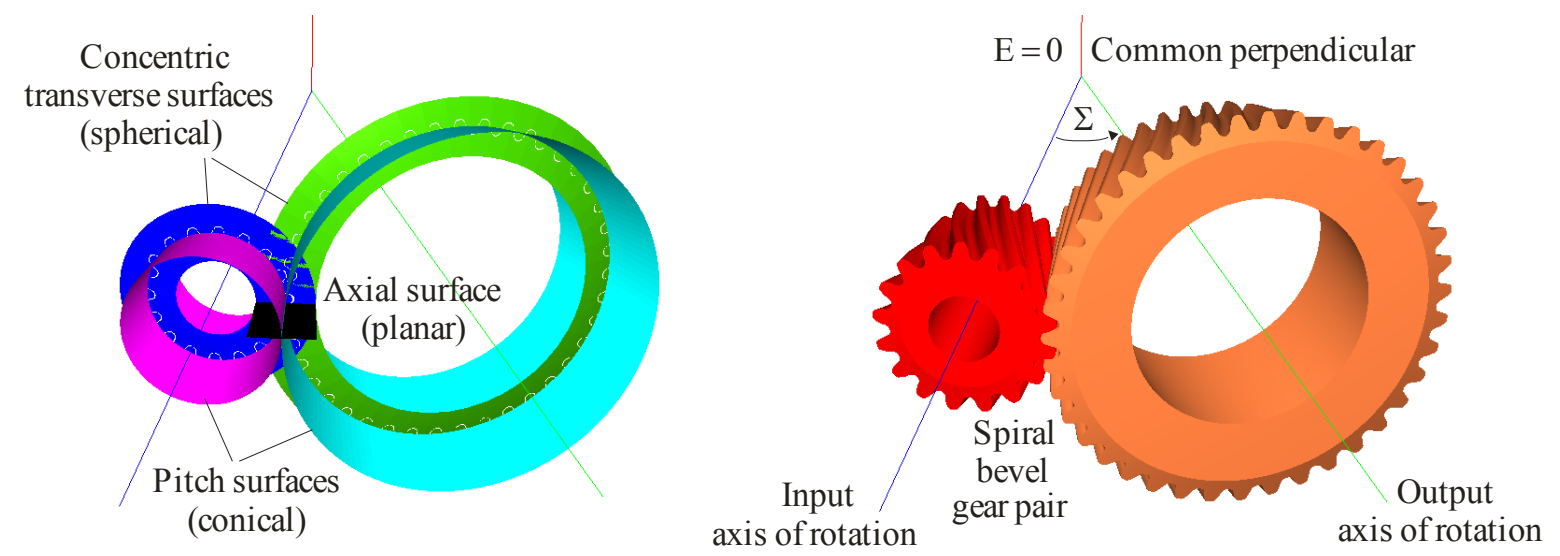

(c) Intersecting axes for input and output gears (spherical coordinates).

Fig. 2. Systems of cylindroidal coordinates.

Case III involves intersecting axis gearing, also referred to as conical and spherical gears (i.e., straight, spiral bevel, and miter). Intersecting axis gearing is defined by $\Sigma \neq 0^{\circ}$ and $\mathrm{E}=0 \mathrm{~mm}$. For this special case, the transverse surfaces are concentric spherical surfaces with centers that coincide with the intersection of the axes of rotation. The axial surface is a special cylindroid; it is a planar surface coincident with the two intersecting axes of rotation. The pitch surfaces are always conical and coincide with the axodes. Their sizes depend on the speed ratio and are independent of the pressure angle and the spiral angle. The infinitely many spherical transverse surfaces determine the face width of the gear. Illustrated in Fig. 2c are two systems of spherical coordinates, defined by the special case of cylindroidal coordinates defined by $\Sigma=30^{\circ}$ and $\mathrm{E}=0 \mathrm{~mm}$. Also shown in Fig. 2c is a sample gear set based on the special cylindroidal coordinates. The intersection between each gear element and one of its spherical transverse surfaces is superimposed on the displayed spherical transverse surface. 


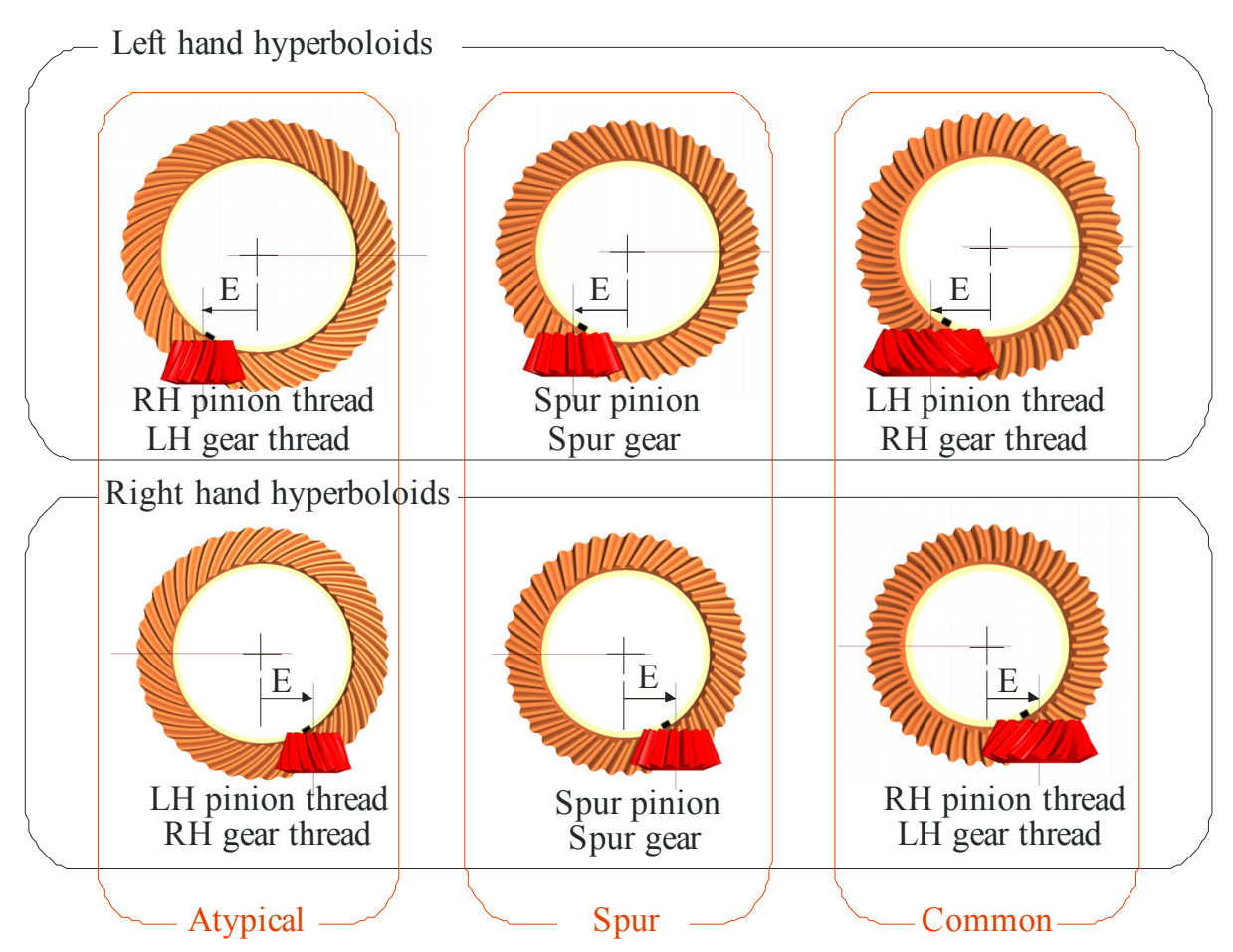

Fig. 3. Hypoid gear sets.

Displayed in Fig. 3 are six variations of a hypoid gear set, each with the same number of teeth (speed ratio), shaft offset, and $90^{\circ}$ shaft angle. It is common gear practice that left-hand (LH) hyperboloids use LH threads on the input gear (pinion) and that right-hand (RH) hyperboloids use RH threads on the input gear. Note that thread hand on one gear element is equal and opposite to the thread hand on its mating gear. For the special case of zero axial contact ratio, both the gear elements are spur. Spur hypoid gear elements experience the minimum relative sliding at the tooth mesh. Atypical is to have LH hyperboloids with RH pinion threads and RH hyperboloids with LH pinion threads. Atypical gear forms illustrate the latent possibilities in establishing a geometric foundation for defining hypoid flank geometry.

\section{Deproximating Tredgold's approximation: hypoid gears}

Presented is gear teeth geometry for generalized spatial gearing in terms of the envelope of a planar tooth profile. This gear flank geometry is similar to spiral bevel gears presented in [1]. The planar tooth profile used in [1] is the classical involute tooth profile. Furthermore, the planar profile was based on a circular pitch curve. The determination of conjugate gear teeth is presented in this article uses a planar rack profile with involute teeth. In other words, the pitch circle of [1] is replaced by a rack with linear flanks. A process for the manufacture of hypoid gears was proposed in [12, 13], using a cutter with variable diameter. This cutter with variable diameter can be modeled as a rack for large spiral angles. A rack is used to simplify the determination of conjugate hypoid flanks. This same procedure can also be used to specify flanks via circular and non-circular auxiliary centrodes (e.g., cycloidal profiles and involute profiles [12, 14]). In either case, deproximating Tredgold's approximation presented here completely removes all approximations resulting in fully conjugate flanks in the axial and transverse directions.

The logic behind the cutter in [1] and modified in this manuscript for a rack profile, is that the planar profile must be positioned in the null plane at each instant in order to determine the envelope of this planar profile. Positioning the planar profile in the null plane to decouple pressure angle from spiral angle, allowing any tooth profile shape can be used (viz., involute, cycloidal, or circular-arc). Mating gear teeth are conjugate as the tooth normal is always reciprocal to the instantaneous screw defined by the two gear rotation axes and the instantaneous gear ratio g (i.e., meshing teeth with zero UTE). This process uses the equivalence of virtual lengths between transverse curves to establish the gear tooth geometry [12].

An overview of cylindroidal coordinates was presented in Section 2. Pitch surfaces consist of a family of co-axial single sheet hyperboloids, where the axis is the axis of rotation. The throats of the hyperboloidal pitch surfaces coincide with the single plane perpendicular to the axis of rotation. Each pitch surface is a ruled surface. The geometry of these ruled surfaces is used to establish the envelope of a planar rack profile. 


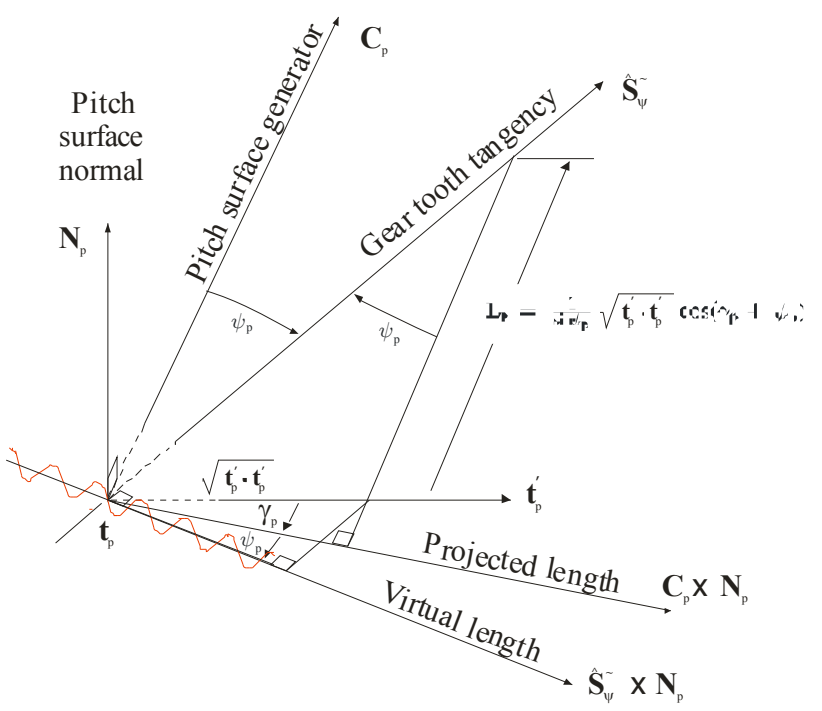

Fig. 4. Tangent plane to pitch surface at point $\mathbf{t}_{p}$.

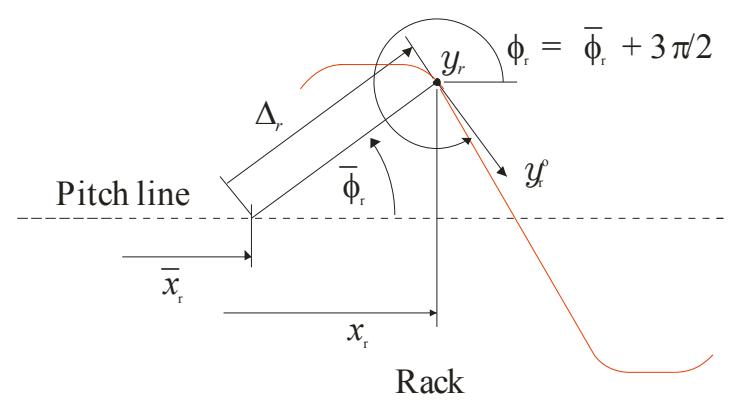

Fig. 5. Normal section of tooth profile.

Depicted in Fig. 4 are an arbitrary point $\mathbf{t}_{p}$ on the ruled hyperboloidal pitch surface, a pitch surface generator direction $\mathbf{C}_{p}$, an instantaneous spiral angle $\psi_{p}$, the lead $\mathrm{L}_{p}$, and a pitch surface normal $\mathbf{N}_{p}$. Perpendicular to gear tooth tangency is a planar gear rack. Depicted in Fig. 5 is a normal section of the gear tooth and a normal section of the planar rack profile. The illustrated profile is a standard involute profile with a pressure angle of $20^{\circ}$. Shown are the coordinates $\left(x_{r}, y_{r}\right)$ for an arbitrary point on the tooth rack. The envelope of $\left(x_{r}, y_{r}\right)$ is obtained by determining the image point $\left(\bar{x}_{r}, \bar{y}_{r}\right)$, created by the intersection of the line of force/action (perpendicular to the tooth surface) with the $\mathrm{x}$-axis. The $\mathrm{x}-$ axis is defined to go through the pitch point of the rack. Solving for the position $\bar{x}_{r}$ yields

$$
\bar{x}_{r}=x_{r}+y_{r} y_{r}^{\circ}
$$

with $y_{r}^{\circ} \equiv d y_{r} / d x_{r}$. The linear position $\bar{x}_{r}$ oscillates about $x_{r}$ as illustrated in Fig. 6 . This means that $\bar{x}_{r}$ can be greater than, less than, or equal to $x_{r}$. The relation between the rack linear position $x_{r}$ and gear rotation angle $\bar{v}_{i}$ is based on the concept of equivalent virtual lengths ${ }^{1}$ :

$$
\bar{x}_{r}=\sqrt{\mathbf{t}_{p}^{\prime} \cdot \mathbf{t}_{p}^{\prime}} \cos \left(\gamma_{p}+\psi_{p}\right)\left(\bar{v}_{i}+\Delta v_{i}\right) .
$$

Here, $\Delta v_{i}$ is the angular shift in the start angle of the tooth spiral for each axial position (i.e., $\Delta v_{i}=w_{i} / \mathrm{L}_{p}$ ). The instantaneous pressure angle $\bar{\phi}_{r}$ of the rack tooth can be expressed

$$
\bar{\phi}_{r}=\tan ^{-1} y_{r}^{\circ}-\frac{3 \pi}{2} .
$$

The chordal distance $\Delta_{r}$ between the contact $\left(x_{r}, y_{r}\right)$ and its pitch point $\left(\bar{x}_{r}, \bar{y}_{r}\right)$ is

${ }^{1}$ For hypoid gears with constant speed ratio g,

$\sqrt{\mathbf{t}_{p}^{\prime} \cdot \mathbf{t}_{p}^{\prime}}=\sqrt{u_{p}^{2}+w_{p}^{2} \sin ^{2} \alpha_{p}}$. 


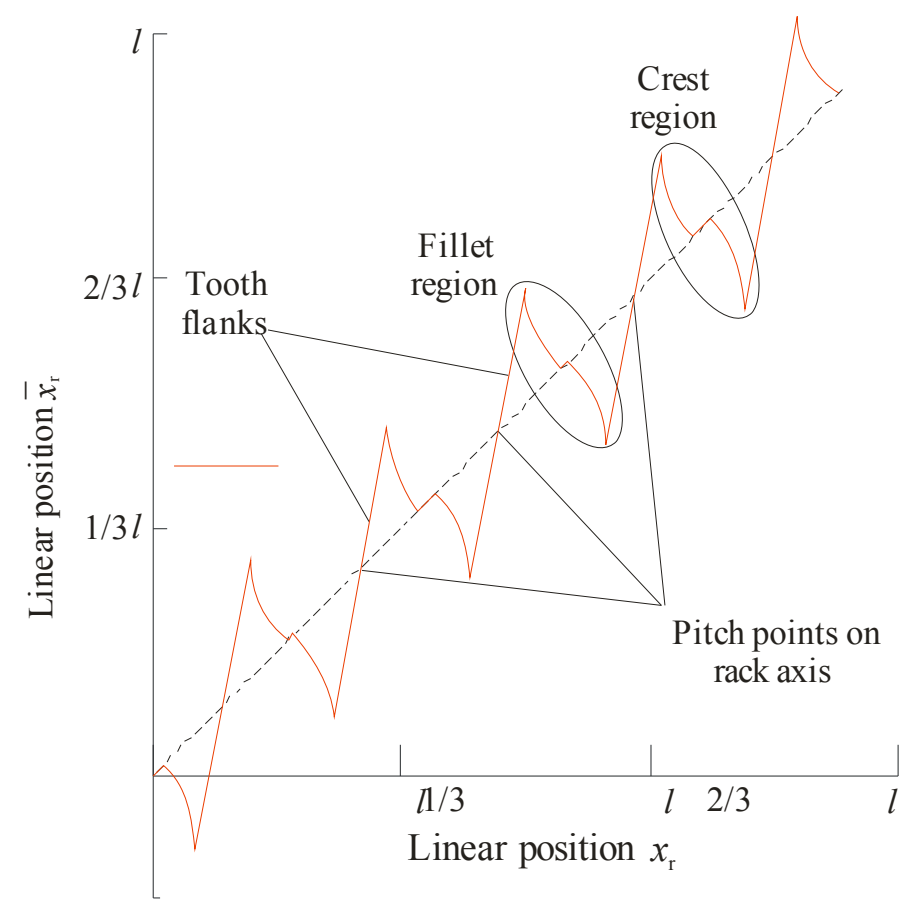

Fig. 6. Relation between $x_{r}$ and $\bar{x}_{r}$.

$$
\Delta_{r}=y_{r} \sqrt{1+y_{r}^{\circ 2}} .
$$

Note that $\Delta_{r}$ changes \pm sign between dedendum and addendum.

Each linear position $x_{r}$ of the planar rack profile defines an image position $\bar{x}_{r}$, an instantaneous pressure angle $\bar{\phi}_{r}$, and a distance $\Delta_{r}$. The position $x_{r}$ depends on the angular position $v_{i}$ of the gear element. Finally, the gear tooth surfaces can be defined through means of conjugate profile traces, which are formulated in Cartesian coordinates. Such coordinates depend on a general point $\mathbf{t}_{p}$ on the hyperboloidal pitch surface along with the pitch surface normal $\mathbf{N}_{p}$ and the spiral tangency $\mathbb{S}_{\psi}^{\sim}$ coincident with $\mathbf{t}_{p}$. The spatial coordinates $\boldsymbol{\Gamma}$ of points on the gear tooth surface are

$$
\boldsymbol{\Gamma}=\mathbf{t}_{p}+\Delta_{r} \boldsymbol{n}
$$

where $\boldsymbol{n}$ is the unit gear tooth flank surface normal. Introducing the direction $\mathbf{B}$ perpendicular to $\mathbb{S}_{\psi}^{\sim}$ and tangent to the pitch surface,

$$
\mathbf{B}=\left(\mathbf{C}_{p} \times \mathbf{N}_{p}\right) \cos \psi_{p}-\mathbf{C}_{p} \sin \psi_{p}
$$

and $n$ becomes

$$
\boldsymbol{n}=\mathbf{B} \cos \bar{\phi}_{r}+\mathbf{N}_{p} \sin \bar{\phi}_{r} .
$$

Using (5) to describe gear teeth, the position and orientation of the polar plane change as $\mathbf{t}_{p}$ changes. Identical results are presented in [1].

The determination of hypoid tooth gear flanks is analogous to the specification of cylindrical gear tooth flanks. Each angular position $v_{i}$ results in a value for gear tooth coordinates $\boldsymbol{\Gamma}$. As the angular position $v_{i}$ varies, $\boldsymbol{\Gamma}$ defines a curve (coordinate curve) that is embedded into the gear tooth surface. The process is repeated for different axial positions $w_{i}$ to establish the entire gear tooth surface (a family of curves or traces). These traces are re-sampled for generating CAD models. First order reciprocity between the gear flank normal and the instantaneous twist defined by the gear rotation axes and speed ratio is invoked to establish fully conjugate flanks. This condition is based on infinitesimal displacements in the transverse and axial directions as defined using the cylindoridal presented in Section 2. 


\section{First order reciprocity}

The concepts of penetration and higher order reciprocity are expanded in this section (see $[2,17])$. Below is a limitation for hypoid gears:

If the nominal pressure angle is constant in the axial direction, it cannot be constant in the transverse

direction. Conversely, if the pressure angle is constant in the transverse direction, it cannot be constant in

the axial direction.

Using an approach similar to [2] based on first order reciprocity, ideally conjugate hypoid tooth geometry across the entire gear flank is obtained by allowing an axial variation in the normal pressure angle with a constant transverse pressure angle. A practical aspect of this feature is that an axial variation in pressure angle facilitates a hobbing type process with a straight sided rack for the fabrication of hypoid gear elements [12,13]. The following determination of $\phi$ neglects transverse and axial profile modifications (i.e., lead crown and tip/roof relief).

The physical tooth flank and the virtual pitch surface intersect along the tooth spiral $\mathbb{S}_{\psi}$. Normal curvature $\kappa_{\psi}$ and geodesic torsion $\tau_{\psi}$ for the tooth flank in the $\mathbb{S}_{\psi}^{\sim}$ direction are expressed [2]

$$
\begin{aligned}
& \kappa_{\psi}=\mathbb{K}_{\psi} \cdot \boldsymbol{n} \\
& \tau_{\psi}=\sqrt{\mathbb{T}_{\psi} \cdot \mathbb{T}_{\psi}}+\Phi_{\psi}^{\dagger}
\end{aligned}
$$

where $\mathbb{K}_{\psi}$ is the curvature vector of $\mathbb{S}_{\psi}, \mathbb{T}_{\psi}$ is the torsion vector of $\mathbb{S}_{\psi}, \Phi_{\psi}$ is the angle between $\boldsymbol{n}$ and $\mathbb{K}_{\psi}$, and $\Phi_{\psi}^{\dagger}$ is the change in $\Phi_{\psi}$ per arc-length $d s_{\lambda}$ along $\mathbb{S}_{\psi}$. Introducing subscripts " $i$ " and " $o$ " to reference the input and output gear elements respectively, the relative normal curvature $\Delta \kappa_{\psi}$ between gear flanks in the spiral direction is

$$
\Delta \kappa_{\psi}=\kappa_{\psi o}-\kappa_{\psi i}
$$

and the relative geodesic torsion $\Delta \tau_{\psi}$ in the spiral direction is

$$
\Delta \tau_{\psi}=\tau_{\psi o}-\tau_{\psi i} .
$$

$\Delta \tau_{\psi}$ is now expanded in terms of $\Phi_{\psi}^{\dagger}$.

$\Phi_{\psi}$ is determined using the inverse tangent function ${ }^{2}$ :

$$
\Phi_{\psi}=\tan ^{-1}\left(\frac{\boldsymbol{n} \cdot \widehat{\mathbb{S}}_{\psi}^{\sim} \times \widehat{\mathbb{K}}_{\psi}}{\boldsymbol{n} \cdot \widehat{\mathbb{K}}_{\psi}}\right)
$$

The differential arc-length $d s_{\lambda}$ for the tooth spiral embedded in the pitch surface is defined in terms of the first fundamental form:

$$
d s_{\lambda} \equiv \sqrt{I_{\lambda}}=\sqrt{\mathcal{E}_{\lambda} d w^{2}+2 \mathcal{F}_{\lambda} d w d v+\mathcal{G}_{\lambda} d v^{2}}
$$

where

$$
\begin{aligned}
\mathcal{E}_{\lambda} & =\mathbf{C}_{p} \cdot \mathbf{C}_{p} \\
\mathcal{F}_{\lambda} & =\mathbf{C}_{p} \cdot \mathbf{t}_{p}^{\prime} \\
\mathcal{G}_{\lambda} & =\mathbf{t}_{p}^{\prime} \cdot \mathbf{t}_{p}^{\prime} .
\end{aligned}
$$

Differentiating (10) with respect to $d s_{\lambda}$,

$$
\begin{aligned}
& \Phi_{\psi}^{\dagger} \equiv \frac{d \Phi_{\psi}}{d s_{\lambda}}= \frac{\left(\boldsymbol{n} \cdot \widehat{\mathbb{K}}_{\psi}\right)\left(\boldsymbol{n}_{\psi}^{\dagger} \cdot \widehat{\mathbb{S}}_{\psi}^{\sim} \times \widehat{\mathbb{K}}_{\psi}+\boldsymbol{n} \cdot \widehat{\mathbb{S}}_{\psi}^{\sim} \times \widehat{\mathbb{K}}_{\psi}^{\dagger}\right)}{\left(\boldsymbol{n} \cdot \widehat{\mathbb{K}}_{\psi}\right)^{2}+\left(\boldsymbol{n} \cdot \widehat{\mathbb{S}}_{\psi}^{\sim} \times \widehat{\mathbb{K}}_{\psi}\right)^{2}}- \\
& \frac{\left(\boldsymbol{n} \cdot \widehat{\mathbb{S}}_{\psi}^{\sim} \times \widehat{\mathbb{K}}_{\psi}\right)\left(\boldsymbol{n}_{\psi}^{\dagger} \cdot \widehat{\mathbb{K}}_{\psi}+\boldsymbol{n} \cdot \widehat{\mathbb{K}}_{\psi}^{\dagger}\right)}{\left(\boldsymbol{n} \cdot \widehat{\mathbb{K}}_{\psi}\right)^{2}+\left(\boldsymbol{n} \cdot \widehat{\mathbb{S}}_{\psi}^{\sim} \times \widehat{\mathbb{K}}_{\psi}\right)^{2}}
\end{aligned}
$$

where $\boldsymbol{n}_{\psi}^{\dagger}$ is the variation in flank unit normal $\boldsymbol{n}$ in the spiral direction $\mathbb{S}_{\psi}^{\sim}$ w.r.t. arc-length $d s_{\lambda}$ for the hyperboloidal pitch surface. $\boldsymbol{n}$ and $\widehat{\mathbb{K}}_{\psi}$ are perpendicular to $\widehat{\mathbb{S}}_{\psi}^{\sim}$ and $\Phi_{\psi}^{\dagger}$ is independent of the normal pressure angle and direction of

${ }^{2} \Phi_{\psi}$ is defined as a positive rotation about the spiral tangent $\mathbb{S}_{\psi}^{\sim}$ from spiral curvature $\mathbb{K}_{\psi}$ to flank normal $\boldsymbol{n}$. 
rotation of the gear elements (viz., drive and coast sides) yet depends on the axial variation in normal pressure angle. As a result, $\tau_{\psi}$ is independent of the normal pressure angle and direction of rotation of the gear elements and independent of the axial variation in normal pressure angle. Conversely, $\kappa_{\psi}$ depends on both pressure angle and direction of rotation of the gear elements. The derivative of $\widehat{\mathbb{K}}_{\psi}$ w.r.t. $d s_{\lambda}$ is presented next.

It is important to recall that $\sqrt{\mathbb{K}_{\psi} \cdot \mathbb{K}_{\psi}}$ is the curvature of the tooth spiral and $\kappa_{\psi}$ is the normal curvature of the tooth flank in the $\mathbb{S}_{\psi}^{\sim}$ direction, see Eq. (6). Likewise, $\sqrt{\mathbb{T}_{\psi} \cdot \mathbb{T}_{\psi}}$ is the torsion of the tooth spiral and $\tau_{\psi}$ is the geodesic torsion of the tooth flank in the $\mathbb{S}_{\psi}^{\sim}$ direction, see Eq. (7). The derivative $\widehat{\mathbb{K}}_{\psi}^{\dagger}$ is expressed in terms of curvature $\mathbb{K}_{\psi}$ and torsion $\mathbb{T}_{\psi}$ accordingly:

$$
\widehat{\mathbb{K}}_{\psi}^{\dagger}=-\left(\sqrt{\mathbb{K}_{\psi} \cdot \mathbb{K}_{\psi}} \boldsymbol{t}+\sqrt{\mathbb{T}_{\psi} \cdot \mathbb{T}_{\psi}} \boldsymbol{b}\right)
$$

with the following unit tangent $\boldsymbol{t}$ and unit binormal $\boldsymbol{b}$ :

$$
\begin{aligned}
\boldsymbol{t} & =\widehat{\mathbb{S}}_{\psi}^{\sim}=\frac{\lambda \mathbf{t}_{p}^{\prime}+\mathbf{C}_{p}}{\sqrt{\left(\lambda \mathbf{t}_{p}^{\prime}+\mathbf{C}_{p}\right) \cdot\left(\lambda \mathbf{t}_{p}^{\prime}+\mathbf{C}_{p}\right)}} \\
\boldsymbol{b} & =\left(\widehat{\mathbb{S}}_{\psi}^{\sim} \times \widehat{\mathbb{K}}_{\psi}\right)
\end{aligned}
$$

where $\lambda=d v / d w$ is the reciprocal of the lead $\mathrm{L}_{p}[12]$ (viz., $\lambda=1 / \mathrm{L}_{p}$ ). The derivative of $\boldsymbol{n}$ w.r.t. $d s_{\lambda}$ is presented next.

Introducing

$$
I_{\lambda} \equiv \frac{1}{d w^{2}} I_{\lambda}=\mathcal{E}_{\lambda}+2 \mathcal{F}_{\lambda} \lambda+\mathcal{G}_{\lambda} \lambda^{2}
$$

the derivative $\boldsymbol{n}_{\psi}^{\dagger}$ is obtained by differentiating $\boldsymbol{n}$ as follows and dividing $d \boldsymbol{n}$ by $d s_{\lambda}$ :

$$
\boldsymbol{n}_{\psi}^{\dagger} \equiv \frac{d \boldsymbol{n}}{d s_{\lambda}}=\boldsymbol{n}^{\prime} \frac{\lambda}{\sqrt{I_{\lambda}}}+\boldsymbol{n}^{\sim} \frac{1}{\sqrt{I_{\lambda}}},
$$

where $\left(\phi^{\sim} \equiv d \phi / d w\right.$ and $\left.\psi^{\sim} \equiv d \psi / d w\right)$

$$
\begin{aligned}
\boldsymbol{n}^{\prime} \equiv & \frac{\partial \boldsymbol{n}}{\partial v}= \\
\boldsymbol{n}^{\sim} \equiv \frac{\partial \boldsymbol{n}}{\partial w}= & \left(\mathbf{C}_{p}^{\prime} \times \mathbf{N}_{p}+\mathbf{C}_{p} \times \mathbf{N}_{p}^{\prime}\right) \cos \psi \cos \phi+\mathbf{N}_{p}^{\sim} \sin \phi-\psi^{\sim}\left[\left(\mathbf{C}_{p} \times \mathbf{N}_{p}\right) \sin \psi+\mathbf{C}_{p} \cos \psi\right] \cos \phi+ \\
& \phi^{\sim}\left[\mathbf{N}_{p} \cos \phi-\mathbf{B} \sin \phi\right] .
\end{aligned}
$$

The above results are combined with (13) to determine $\Phi_{\psi}^{\dagger}$ and then $\tau_{\psi}$ of (7). $\Delta \kappa_{\psi}$ and $\Delta \tau_{\psi}$ are combined to establish zero Gaussian curvature between gear flanks.

A relative curvature $\Delta \kappa$ exists for each direction tangent to the gear flanks. These relative curvatures exist for each axial position between the gear flanks. $\Delta \kappa$ must always be positive to avoid interference or penetration between meshing flanks. Interference is avoided if there exists an axial variation in the normal pressure angle $\phi$. In [2], $\Delta \kappa$ was used for constant $\phi$ in the transverse and axial directions and $\Delta \kappa_{*}$ was used for constant $\phi$ in the axial direction (viz., $\phi^{\prime} \neq 0$ and $\phi^{\sim}=0$ ). Here, $\Delta \kappa_{*}$ is the relative curvature for $\phi^{\prime}=0$ and $\phi^{\sim} \neq 0$ where $\max \Delta \kappa_{*}$ and ${ }_{\text {min }} \Delta \kappa_{*}$ are the extreme relative curvatures between meshing gear flanks. From [2], $\Delta \kappa_{*}$ is zero in the $\bar{\eta}_{*}$ direction where ${ }^{3}$

$$
\begin{aligned}
& \tan \bar{\eta}_{*}=-\frac{\Delta \kappa_{\psi}}{\Delta \tau_{\psi}} \\
& \min _{\min }=0 \\
& \kappa_{\max } \Delta \kappa_{*}=\frac{\Delta \kappa_{\psi}^{2}+\Delta \tau_{\psi}^{2}}{\Delta \kappa_{\psi}}=\frac{\Delta \kappa_{\psi}}{\sin ^{2} \bar{\eta}_{*}} .
\end{aligned}
$$

The objective is to determine $\phi^{\sim}$ and subsequently $\phi$ for first order reciprocity $\left(\phi_{i}^{\sim}=\phi_{o}^{\sim}\right)$.

${ }^{3} \sin ^{2} \bar{\eta}_{*}$ and $\cos ^{2} \bar{\eta}_{*}$ are obtained from $\tan \bar{\eta}_{*}$ as follows:

$\sin ^{2} \bar{\eta}_{*}=\frac{\Delta \kappa_{\psi}^{2}}{\Delta \kappa_{\psi}^{2}+\Delta \tau_{\psi}^{2}}$ and $\cos ^{2} \bar{\eta}_{*}=\frac{\Delta \tau_{\psi}^{2}}{\Delta \kappa_{\psi}^{2}+\Delta \tau_{\psi}^{2}}$. 
Each gear flank is defined parametrically in terms of coordinate curves $\mathbb{S}_{\psi}$ and $\boldsymbol{\Gamma}$ using cylindroidal coordinates $(v, w)$. From methods of classical differential geometry $[2,5]$, the normal curvature $\kappa$ at a point on the gear flank is

$$
\kappa=\frac{e+2 f \beta+g \beta^{2}}{\mathcal{E}+2 \mathcal{F} \beta+\mathcal{G} \beta^{2}}=\frac{I I}{I}
$$

where $\beta=d v / d w$ and

$$
\begin{aligned}
e & =\mathbb{S}_{\psi}^{\sim} \cdot \boldsymbol{n} \\
f & =\boldsymbol{\Gamma}^{\prime \sim} \cdot \boldsymbol{n} \\
g & =\boldsymbol{\Gamma}^{\prime \prime} \cdot \boldsymbol{n} \\
\mathcal{E} & =\mathbb{S}_{\psi}^{\sim} \cdot \mathbb{S}_{\psi}^{\sim} \\
\mathcal{F} & =\mathbb{S}_{\psi}^{\sim} \cdot \boldsymbol{\Gamma}^{\prime} \\
\mathcal{G} & =\boldsymbol{\Gamma}^{\prime} \cdot \boldsymbol{\Gamma}^{\prime} .
\end{aligned}
$$

In general, $\mathbb{S}_{\psi}$ and $\boldsymbol{\Gamma}$ are not geodesics. Curvature is defined in terms of $\beta$. Next, $\beta$ is expressed in terms of an angle $\theta$, a CCW rotation about the outward surface normal $\boldsymbol{n}$ from $\mathbb{S}_{\psi}^{\sim}$.

The tangent $\mathbf{T}$ to the gear tooth surface is

$$
\mathbf{T}=\mathbb{S}_{\psi}^{\sim} d w+\Gamma^{\prime} d v
$$

$\mathbf{T}$ is specified in terms of $\theta$. The differential arc-length $d s$ for $\mathbf{T}$ is defined in terms of the first fundamental form $I$ :

$$
d s \equiv \sqrt{I}=\sqrt{\mathcal{E} d w^{2}+2 \mathcal{F} d w d v+\mathcal{G} d v^{2}} .
$$

Note that $d s$ is for the tooth flank and $d s_{\lambda}$ is for the tooth spiral on the pitch surface. Moreover, $d v / d w$ of (11) is between $\mathbf{t}_{p}^{\prime}$ and $\mathbf{C}_{p}$ of the pitch surface whereas $d v / d w$ of (21) is between $\boldsymbol{\Gamma}^{\prime}$ and $\mathbb{S}_{\psi}^{\sim}$ of the tooth flank. The ratio $\beta$ is obtained via the following vector dot product:

$$
\mathbf{T} \cdot \mathbb{S}_{\psi}^{\sim}=\sqrt{I} \sqrt{\mathbb{S}_{\psi} \cdot \mathbb{S}_{\psi}} \cos \theta
$$

where

$$
\mathbf{T} \cdot \mathbb{S}_{\psi}^{\sim}=\mathbb{S}_{\psi}^{\sim} \cdot \mathbb{S}_{\psi}^{\sim} d w+\mathbf{\Gamma}^{\prime} \cdot \mathbb{S}_{\psi}^{\sim} d v .
$$

Expanding above gives the following quadratic:

$$
A_{\theta} \beta^{2}+B_{\theta} \beta+C_{\theta}=0
$$

where

$$
\beta=\frac{-B_{\theta} \pm \sqrt{B_{\theta}^{2}-4 A_{\theta} C_{\theta}}}{2 A_{\theta}},
$$

and

$$
\begin{aligned}
A_{\theta} & =\mathcal{F}^{2}-\mathcal{E} \mathcal{G} \cos ^{2} \theta \\
B_{\theta} & =-2 \mathcal{E} \mathcal{F} \sin ^{2} \theta \\
C_{\theta} & =\mathcal{E}^{2} \sin ^{2} \theta
\end{aligned}
$$

Gear flanks are tangent in the direction $\theta=\bar{\eta}_{*}$.

Tangency in the direction of zero relative curvature ensures first order reciprocity between flanks [2]. From (17),

$$
\kappa *_{i}\left(\theta=\bar{\eta}_{*}\right)=\kappa *_{o}\left(\theta=\bar{\eta}_{*}\right) .
$$

Zero relative curvature coincides with minimum relative curvature for tangency in the $\theta=\bar{\eta}_{*}$ direction. First order reciprocity can also be obtained by specifying zero relative geodesic torsion for $\theta=\bar{\eta}_{*}$ where geodesic torsion $\tau=0.5$ $d \kappa / d \theta$ and $\beta(\theta)$ [2]. Using (19) to express normal curvature, above becomes ${ }^{4}$

$$
f\left(\phi^{\sim}(w, \phi)\right)=\left(e_{i}+2 f_{i} \beta_{i}+g_{i} \beta_{i}^{2}\right) I_{o}-\left(e_{o}+2 f_{o} \beta_{o}+g_{o} \beta_{o}^{2}\right) I_{i} .
$$

${ }^{4}$ Note that $f$ represents a function whereas $f_{i}$ and $f_{o}$ are coefficients. 
The axial variation in normal pressure angle $\phi^{\sim}$ exists within the second fundamental form coefficients $f_{i}$ and $f_{o}$ together with ratios $\beta_{i}$ and $\beta_{o}$.

$\phi^{\sim}$ is expressed implicitly (i.e., not explicitly) and an immediate solution to (26) is not recognized. As a result, numerical root-finding methods (viz., secant method) are used to obtain $\phi^{\sim}$. Subsequently $\phi$ is obtained using numerical differential equation solving techniques (viz., Runge-Kutta). (26) can be simplified for the case of hypoid gears with uniform motion. From (23), two quadratic relations involving the ratio $\beta$ and angle $\bar{\eta}_{*}$ exist for the input tooth flank and the output tooth flank. These two quadratic relations are combined to eliminate $\beta$ via Sylvester's method:

$$
A X=\left(\begin{array}{cccc}
0 & A_{\theta i} & B_{\theta i} & C_{\theta i} \\
0 & A_{\theta o} & B_{\theta o} & C_{\theta o} \\
A_{\theta i} & B_{\theta i} & C_{\theta i} & 0 \\
A_{\theta o} & B_{\theta o} & C_{\theta o} & 0
\end{array}\right)\left(\begin{array}{c}
\beta^{3} \\
\beta^{2} \\
\beta \\
1
\end{array}\right)=0 .
$$

Expanding det $A=0$ results in a special quartic polynomial with no odd powers. Solving for $\sin ^{2} \bar{\eta}_{*}$ gives

$$
\sin ^{2} \bar{\eta}_{*}=\frac{2 A_{\bar{\eta}_{*}}}{B_{\bar{\eta}_{*}} \pm \sqrt{B_{\bar{\eta}_{*}}^{2}-4 A_{\bar{\eta}_{*}} C_{\bar{\eta}_{*}}}},
$$

where

$$
\begin{aligned}
A_{\bar{\eta}_{*}}= & 2 \mathcal{E}_{i}^{2} \mathcal{E}_{o}^{2}\left[\left(\mathcal{F}_{i}^{2}-\mathcal{E}_{i} \mathcal{G}_{i}\right)\left(\mathcal{F}_{o}^{2}-\mathcal{E}_{o} \mathcal{G}_{o}\right)\right]-\left[\mathcal{E}_{o}^{4}\left(\mathcal{F}_{i}^{2}-\mathcal{E}_{i} \mathcal{G}_{i}\right)^{2}+\mathcal{E}_{i}^{4}\left(\mathcal{F}_{o}^{2}-\mathcal{E}_{o} \mathcal{G}_{o}\right)^{2}\right] \\
B_{\bar{\eta}_{*}}= & 2\left[\mathcal{E}_{i}^{4}\left(\mathcal{F}_{o}^{2}-\mathcal{E}_{o} \mathcal{G}_{o}\right) \mathcal{E}_{o} \mathcal{G}_{o}+\mathcal{E}_{o}^{4}\left(\mathcal{F}_{i}^{2}-\mathcal{E}_{i} \mathcal{G}_{i}\right) \mathcal{E}_{i} \mathcal{G}_{i}\right]+ \\
& 4 \mathcal{E}_{i}^{2} \mathcal{E}_{o}^{2}\left[\mathcal{F}_{o}^{2}\left(\mathcal{F}_{i}^{2}-\mathcal{E}_{i} \mathcal{G}_{i}\right)+\mathcal{F}_{i}^{2}\left(\mathcal{F}_{o}^{2}-\mathcal{E}_{o} \mathcal{G}_{o}\right)\right]+ \\
& 4 \mathcal{E}_{i} \mathcal{E}_{o} \mathcal{F}_{i} \mathcal{F}_{o}\left[\mathcal{E}_{o}^{2}\left(\mathcal{F}_{i}^{2}-\mathcal{E}_{i} \mathcal{G}_{i}\right)+\mathcal{E}_{i}^{2}\left(\mathcal{F}_{o}^{2}-\mathcal{E}_{o} \mathcal{G}_{o}\right)\right]- \\
& 2 \mathcal{E}_{i}^{2} \mathcal{E}_{o}^{2}\left[\left(\mathcal{F}_{i}^{2}-\mathcal{E}_{i} \mathcal{G}_{i}\right) \mathcal{E}_{o} \mathcal{G}_{o}+\left(\mathcal{F}_{o}^{2}-\mathcal{E}_{o} \mathcal{G}_{o}\right) \mathcal{E}_{i} \mathcal{G}_{i}\right] \\
C_{\bar{\eta}_{*}}= & \mathcal{E}_{i}^{2} \mathcal{E}_{o}^{2}\left[2 \mathcal{E}_{i} \mathcal{E}_{o} \mathcal{G}_{i} \mathcal{G}_{o}-\left(\mathcal{E}_{o}^{2} \mathcal{G}_{i}^{2}+\mathcal{E}_{i}^{2} \mathcal{G}_{o}^{2}\right)\right]+4 \mathcal{E}_{i} \mathcal{E}_{o} \mathcal{F}_{i} \mathcal{F}_{o}\left(\mathcal{E}_{o}^{2} \mathcal{E}_{i} \mathcal{G}_{i}+\mathcal{E}_{i}^{2} \mathcal{E}_{o} \mathcal{G}_{o}\right)- \\
& 4 \mathcal{E}_{i}^{2} \mathcal{E}_{o}^{2}\left(\mathcal{E}_{i} \mathcal{G}_{i} \mathcal{F}_{o}^{2}+\mathcal{E}_{o} \mathcal{G}_{o} \mathcal{F}_{i}^{2}\right)
\end{aligned}
$$

Additional simplifications exists for hypoid gears where $\kappa_{i}\left(\theta=\bar{\eta}_{*}\right)=\kappa_{*_{o}}\left(\theta=\bar{\eta}_{*}\right)=0$. Expanding the coefficient $f$ to

$$
f=\boldsymbol{\Gamma}^{\sim} \cdot \boldsymbol{n}=a_{\phi} \phi^{\sim}+b_{\phi}
$$

and recognizing that

$$
e+2 f \beta+g \beta^{2}=0
$$

the axial variation in pressure angle $\phi^{\sim}$ becomes

$$
\phi^{\sim}=-\frac{e+2 b_{\phi} \beta+g \beta^{2}}{2 a_{\phi} \beta},
$$

where $\left(\boldsymbol{n} \cdot \boldsymbol{n}=1, \boldsymbol{n}^{\sim} \cdot \boldsymbol{n}=0\right.$, and $\left.\boldsymbol{n}^{\prime} \cdot \boldsymbol{n}=0\right)$

$$
\begin{aligned}
a_{\phi}= & \frac{1+\cos ^{2} \phi}{\sin ^{3} \phi}\left[\sqrt{\mathbf{t}_{p}^{\prime} \cdot \mathbf{t}_{p}^{\prime}} \cos \left(\gamma_{p}+\psi_{p}\right)\right]-2 \frac{\cos \phi}{\sin ^{3} \phi}\left(\mathbf{t}_{p}^{\prime} \cdot \boldsymbol{n}\right) \\
b_{\phi}= & \frac{1}{\sin ^{2} \phi}\left[\left(\mathbf{t}_{p}^{\prime \prime} \lambda+\mathbf{t}_{p}^{\prime}\right) \cdot \boldsymbol{n}\right]+\frac{\cos \phi}{\sin ^{2} \phi}\left[\sqrt{\mathbf{t}_{p}^{\prime} \cdot \mathbf{t}_{p}^{\prime}} \sin \left(\gamma_{p}+\psi_{p}\right)\left(\gamma_{p}^{\sim}+\psi_{p}^{\sim}\right)\right]- \\
& \frac{\cos \phi}{\sin ^{2} \phi}\left[\frac{\mathbf{t}_{p}^{\prime} \cdot \mathbf{t}_{p}^{\prime} \cos \left(\gamma_{p}+\psi_{p}\right)}{\sqrt{\mathbf{t}_{p}^{\prime} \cdot \mathbf{t}_{p}^{\prime}}}\right] .
\end{aligned}
$$

$\bar{x}_{r}=x_{r}$ at the pitch point. $\bar{\phi}_{r}^{\prime}=\phi_{r}^{\prime}=0$ is specified for zero variation in transverse pressure angle. Also, $\psi^{\prime}=0$ for any value of $\phi_{r}$ (see second law of gearing [12]). An expansion on the coefficients in the first and second fundamental forms are presented in the literature [12,2]. Derivatives of $\boldsymbol{\Gamma}$ are reserved for the reader. A hypoid gear example is presented where $\phi$ is based on the above differential equation. 


\section{Illustrative example}

A quantitative measure on the conjugacy of hypoid tooth flanks is presented. A CAD model of an automotive hypoid gear set was created using the methodology presented in Sections 3 and 4 to describe tooth flanks. The hypoid gear pair was based on a 1999 Ford Mustang coupe $[2,15,16]$. A drop-in replacement design for the OEM hypoid gear pair was created by using the same tooth count and nominal measurements of offset, face width, and pitch diameters. The Mustang came from the factory with a Ford 7.5 inch 2.93 ratio hypoid gear set (i.e., the outside diameter of the output gear element is $7.5 \mathrm{in})$. The macro-geometry of this gear set is the same as $[2,15,16]$ whereas the micro-geometry of the tooth flanks is different. Virtual models of the gear elements in mesh are depicted in Fig. 7.

A summary of the hypoid gear data is presented in Table 2. It is noteworthy that the transverse contact ratio is specified to be 0.85 . The gear set has "left-hand" hyperboloids. Additionally, the spiral on the input gear is left-hand. This left-hand combination of hand and spiral results in tooth flank contact on the concave side of the input tooth and tooth flank contact on the convex side of the mating output gear tooth. Such contact conditions tend to increase backlash when loaded.

It is instructive to investigate the derivative $\phi^{\sim}$ prior to the determination of $\phi$ presented in Section 4 . Depicted in Fig. 8 are graphs of $\phi^{\sim}$ for different values of constant $\phi$. Specifying $\phi$ for an axial position is a boundary condition necessary to determine $\phi$ across the gear flank. Illustrated in Fig. 9 are graphs of $\phi$ where $\phi$ was specified at the throat of the hyperboloidal pitch surfaces. In general, $\phi$ will be specified somewhere on the gear flank (e.g., toe, heel, or midflank) and it is not reasonable to specify $\phi$ at the throat. In all cases, the pressure angle asymptotically approaches a limiting value as the axial position increases. High values of pressure angle $\left(\approx 90^{\circ}\right)$ are not recommended due to high contact loads and low values of pressure angle $\left(\approx 0^{\circ}\right)$ are not recommended due to high values of contact stress. Negative pressure angles can be realized by transposing the addendum and dedendum. Recognition of this behavior is useful when specifying nominal pressure angle. The nominal pressure angle for the example hypoid gear set is specified to be $30^{\circ}$ for both sides of the flank. $30^{\circ}$ was used as the nominal pressure angle and not $20^{\circ}$ used earlier $[2,15,16]$. A nominal value of $20^{\circ}$ results in unacceptable $\phi$ values at the toe. Displayed in Fig. 10 is the normal pressure angle $\phi_{n}$ for clockwise $(\mathrm{CW})$ and counter clockwise $(\mathrm{CCW})$ rotations of the input gear (pinion). Also shown is the angle $\bar{\eta}_{*}$, the inclination of the line of contact relative to the pitch surface. Depicted in Fig. 11 is a gear tooth illustrating a transverse profile and coordinate curve. The transverse profile is the intersection between the tooth and the transverse surface (see Figs. 1 and 2 in Section 2). The flank normals along the coordinate curve $\Gamma$ intersect a single transverse curve (intersection between transverse surface and pitch surface) with a constant pressure angle (see Fig. 1). The flank normals along the contact patch intersect the pitch surface, each with a different pressure angle. Also shown is the tooth spiral.

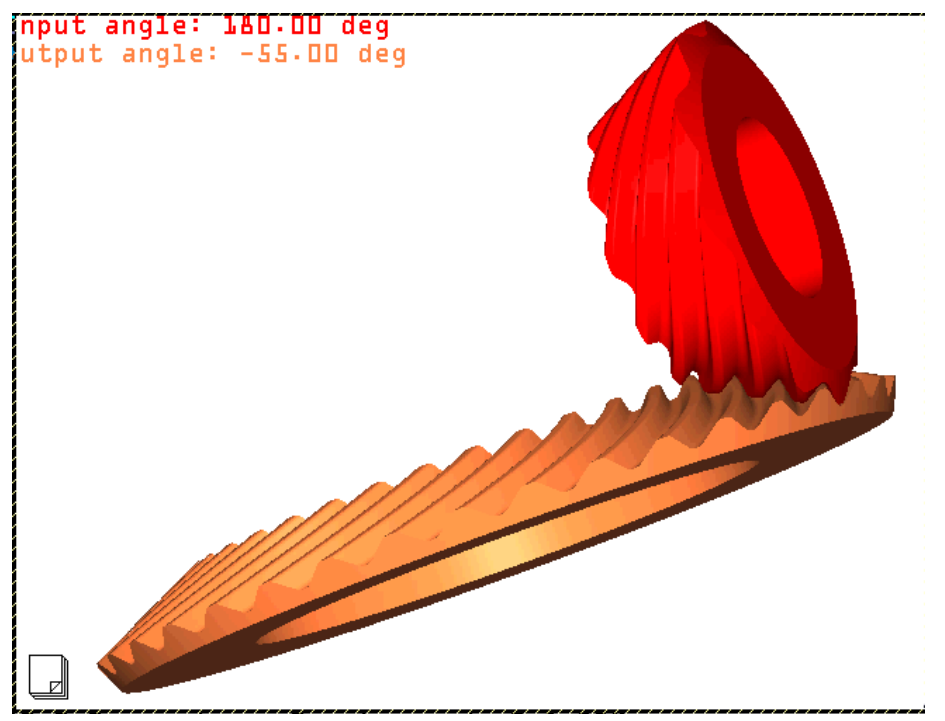

Fig. 7. Hypoid gear pair with contact pattern. 
Table 2. Gear pair data.

\begin{tabular}{|c|c|c|}
\hline Gear data & Input gear & Output gear \\
\hline Number of teeth & 11 & 36 \\
\hline Shaft offset [mm] & \multicolumn{2}{|c|}{25.4} \\
\hline Shaft angle [deg.] & \multicolumn{2}{|c|}{90.00} \\
\hline Mean spiral angle [deg.] & \multicolumn{2}{|c|}{60.84} \\
\hline Face width $[\mathrm{mm}]$ & \multicolumn{2}{|c|}{33.66} \\
\hline Mid-face contact [mm] & \multicolumn{2}{|c|}{89.85} \\
\hline Transverse contact ratio [-] & \multicolumn{2}{|c|}{0.85} \\
\hline Axial contact ratio [-] & \multicolumn{2}{|c|}{3.00} \\
\hline Generator angle [deg.] & 25.415 & 64.585 \\
\hline Nominal pressure angle [deg.] & \multicolumn{2}{|c|}{30} \\
\hline Normal tooth module [mm] & \multicolumn{2}{|c|}{3.118} \\
\hline Addendum (nominal) [mm] & \multicolumn{2}{|c|}{2.650} \\
\hline Dedendum (nominal) [mm] & \multicolumn{2}{|c|}{2.962} \\
\hline Hand of spiral & LH & RH \\
\hline Hand of pitch surface & \multicolumn{2}{|c|}{$\mathrm{LH}$} \\
\hline Transverse relief constant [-] & $0.00 / 0.01$ & $0.00 / 0.01$ \\
\hline Axial relief constant [-] & $0.00 / 0.01$ & $0.00 / 0.01$ \\
\hline
\end{tabular}

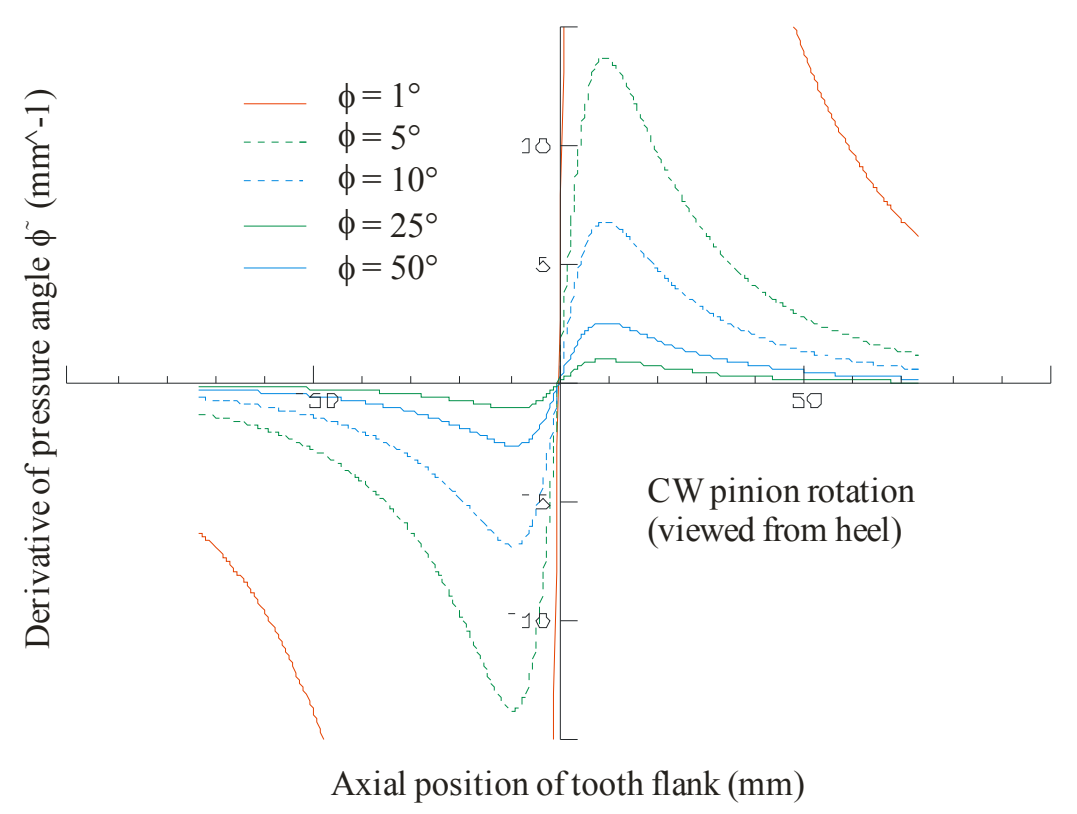

Fig. 8. Axial variation in normal pressure. 


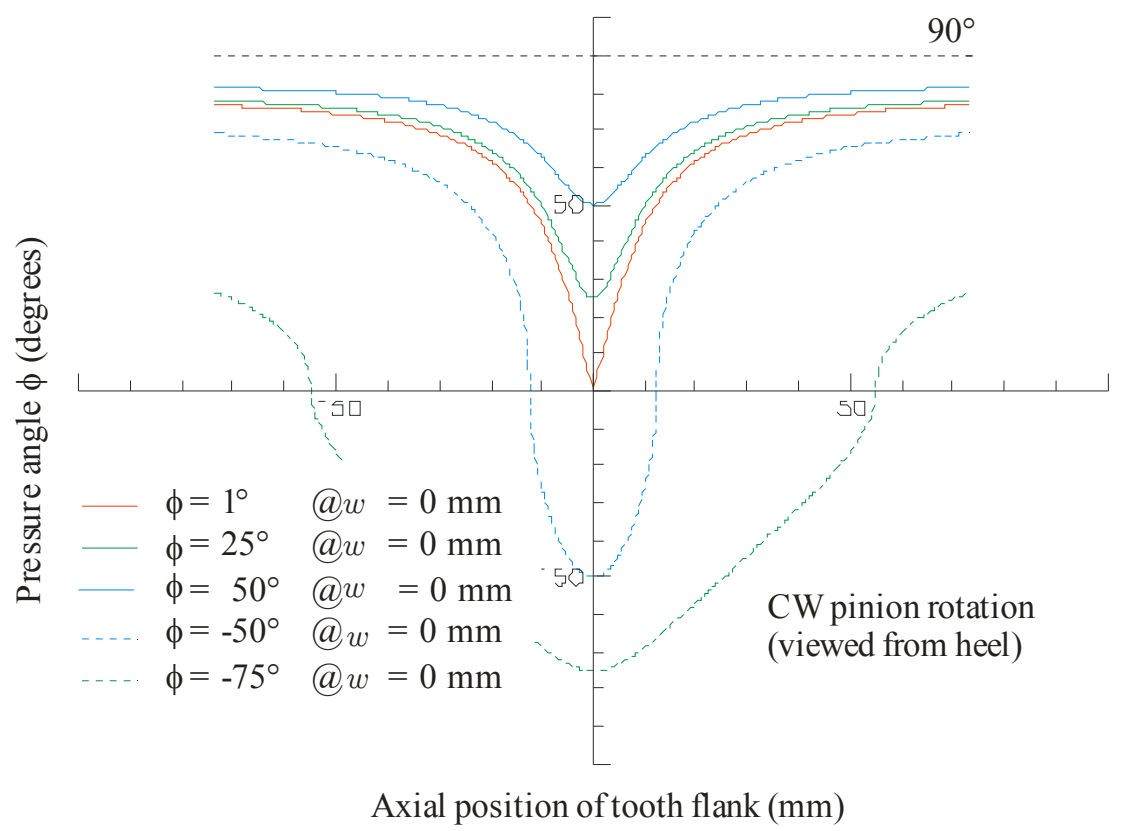

Fig 9. Pressure angle based initial value specified at throat of hyperboloidal pitch surface.

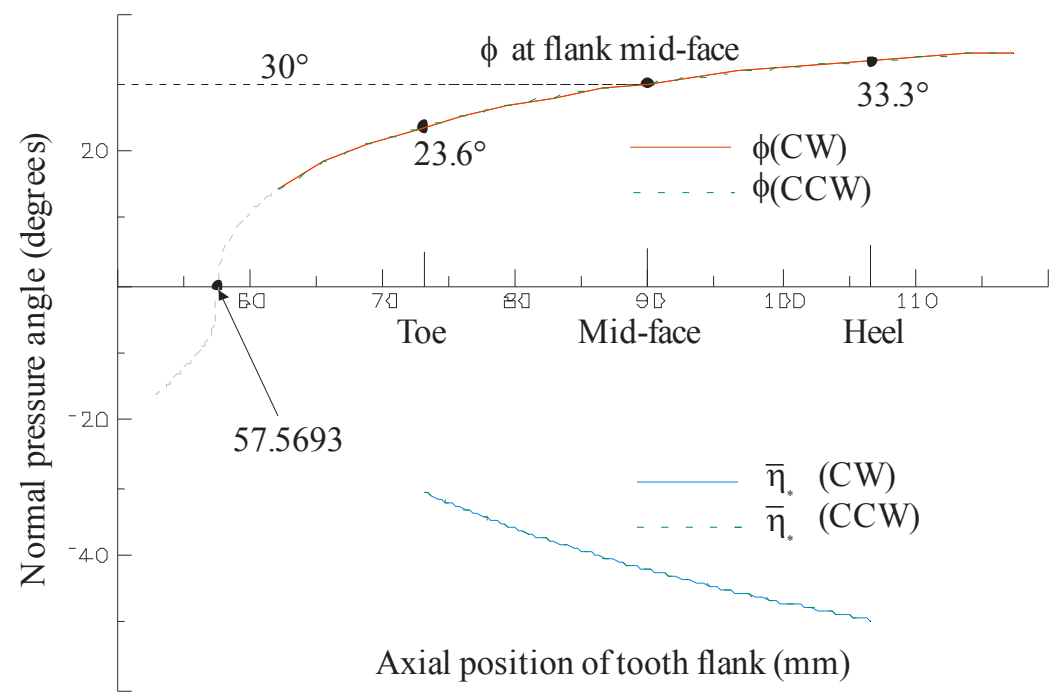

Fig 10. Variation in normal pressure angle.

Transverse profile $(\phi \neq$ constant $)$

Contact patch

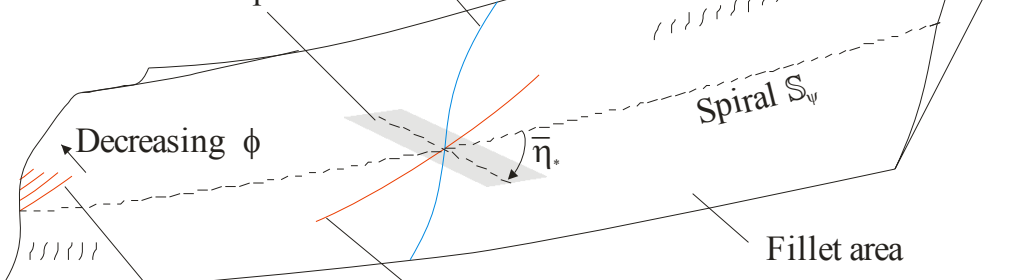

Involute coordinate curve

Involute coordinate curve $\Gamma$

$\left(\phi=23.6^{\circ}\right)$ $\left(\phi=30^{\circ}=\right.$ constant $)$

Fig. 11. Gear tooth with coordinate and transverse curves. 
Enlarged views of the input and output gear teeth in mesh are provided in Fig. 12. Fig. 12a depicts gear mesh for $\mathrm{CW}$ rotation of the input gear (as viewed from the heel or outer diameter) and Fig. 12b depicts gear mesh for CCW rotation of the input gear.

A micro-geometry evaluation of the hypoid gear teeth conjugacy uses the Ease-off based methodology for unloaded tooth contact described in [1]. Illustrated in Fig. 13 is the Ease-off topography of the gear flanks for CW and CCW rotation of the input gear element. The Ease-off topography for CW (drive side) and CCW (coast side) rotation of the input gear element is zero. There exists spikes in the UEO topography graphs. These spikes are not due to numerical illconditioning, they are a result of decreasing values of pressure angle in the toe direction as evidenced in Fig. 9. Fig. 7 also includes a video animation of the mesh for $\mathrm{CW}$ and $\mathrm{CCW}$ rotation of the input gear. Views from the back side of the mesh qualitatively illustrates the contact "evenly" traversing the flanks.

Additional insight into the fully conjugate flanks is revealed by looking at normal curvature and geodesic torsion of the contacting surfaces. Presented in Fig. 14 are graphs of input and output tooth curvatures with constant pressure angle (viz., $\kappa_{i}$ and $\kappa_{o}$ respectively) together with their relative curvature $\Delta \kappa$. Also presented are graphs of input and output tooth curvatures with varying pressure angle (viz., $\kappa_{* i}$ and $\kappa_{* 0}$ respectively) together with their relative curvature $\Delta \kappa_{*}$. As reported in [2], reciprocity is a necessary condition but not sufficient for hypoid tooth contact. In other words, gear flanks with constant pressure can be locally reciprocal with hyperbolic contact. The magnitude of interference in Fig. 14 is "small" $\left(\Delta \kappa \leq 1.0 \mu m^{-1}\right)$. Fig. 15 shows zoomed-in images of the penetration region (negative relative curvature). Interference is maximum at the toe and decreases monotonically towards the heel. The magnitude of the penetration is different for $\mathrm{CW}$ and $\mathrm{CCW}$ rotations of the input gear. Subtle is that $\bar{\eta}_{*}$ is asymmetrically placed on different sides of $\eta$ for $\mathrm{CW}$ and $\mathrm{CCW}$ rotations. Using techniques presented in [2], second order reciprocity exists; namely, $\kappa_{* i}^{\dagger}\left(\bar{\eta}_{*}\right)=\kappa_{* o}^{\dagger}\left(\bar{\eta}_{*}\right)$ and $\tau_{* i}^{\dagger}\left(\bar{\eta}_{*}\right)=\tau_{* o}^{\dagger}\left(\bar{\eta}_{*}\right)$. Together with zero $(\leq 1 \mu \mathrm{m})$ maximum UEO topography, the presented process is capable of generating "fully" conjugate flanks in the axial and transverse directions. General profile modification is presented next.

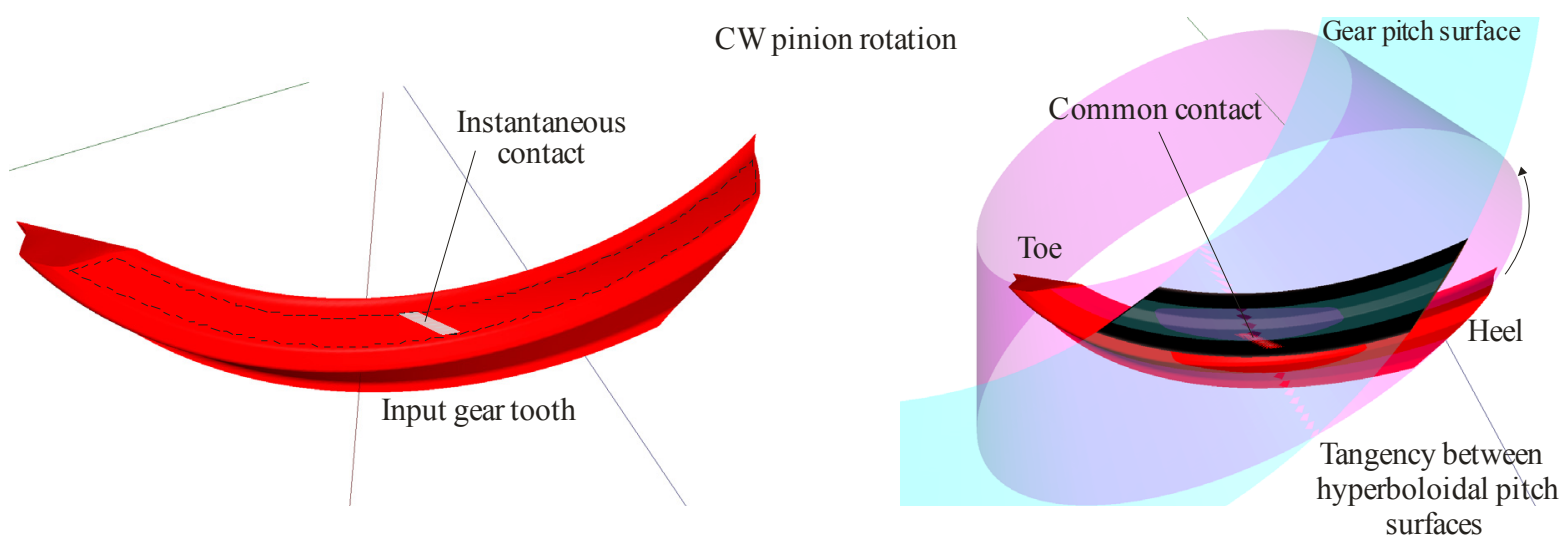

(a) Clockwise rotation of input gear

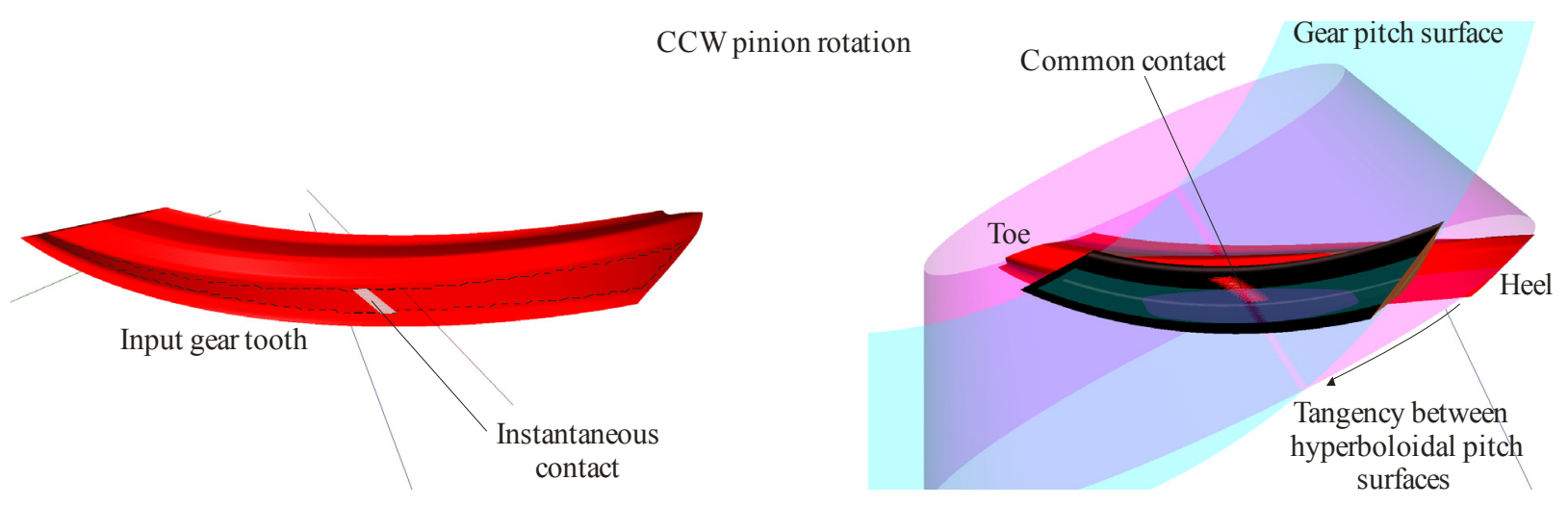

(b) Counter clockwise rotation of input gear.

Fig. 12. Input gear teeth with contact pattern. 


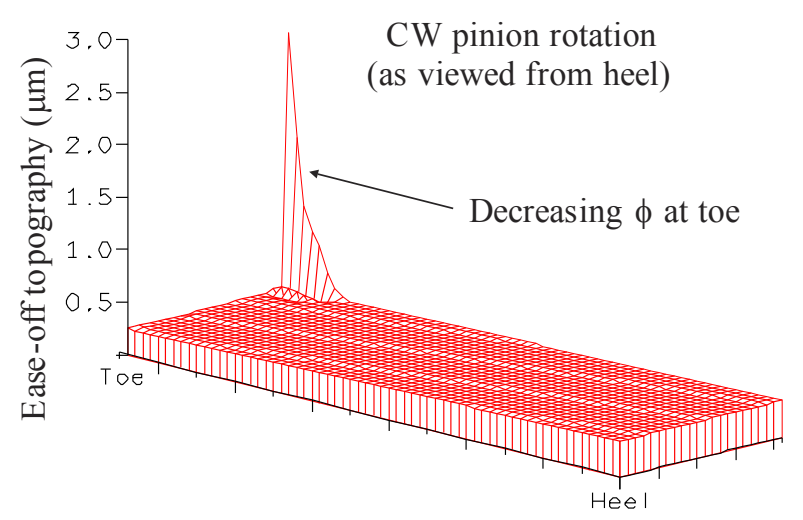

(a) CW rotation of input gear element (left side)

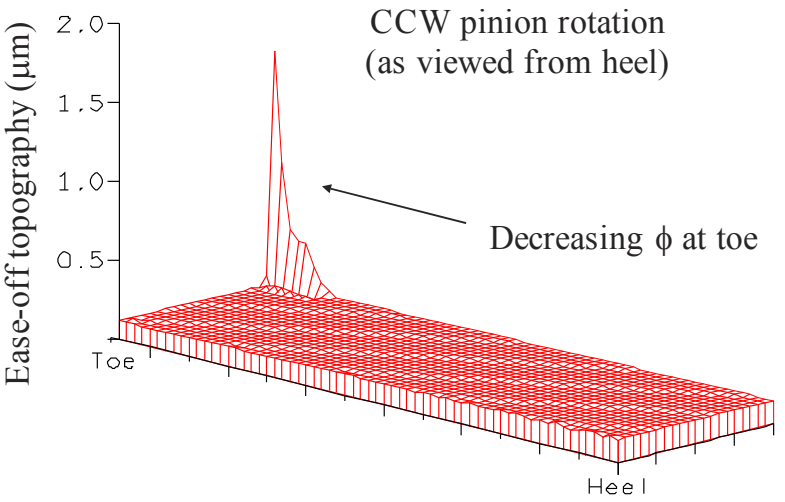

(b) $\mathrm{CCW}$ rotation of gear element (right side)

Fig. 13. Ease-off topography for gear set generated using data in Table 2.

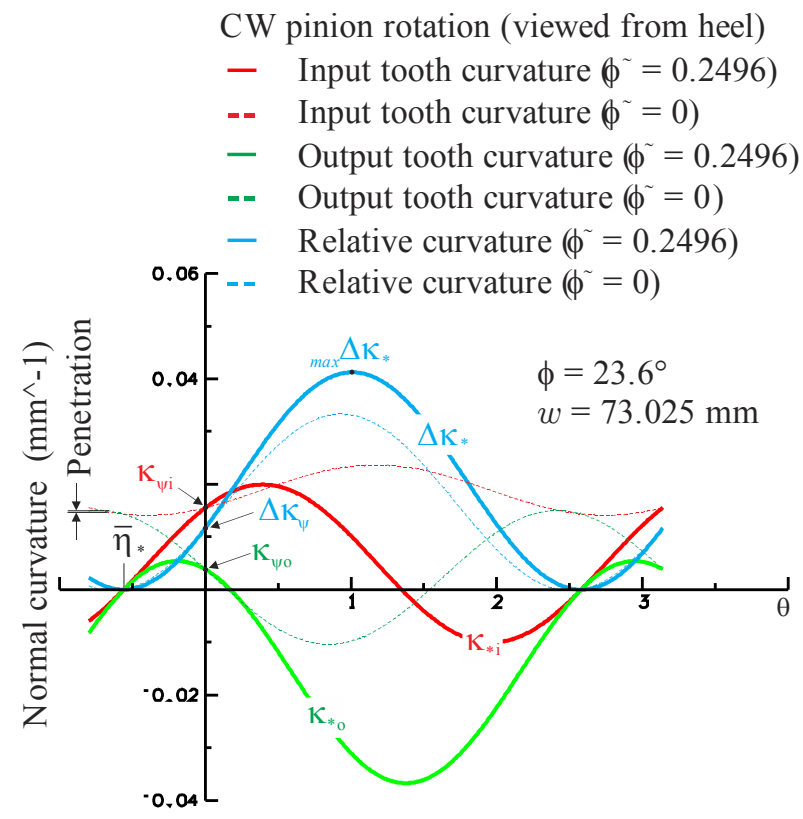

Angle relative to spiral tangent (radians)

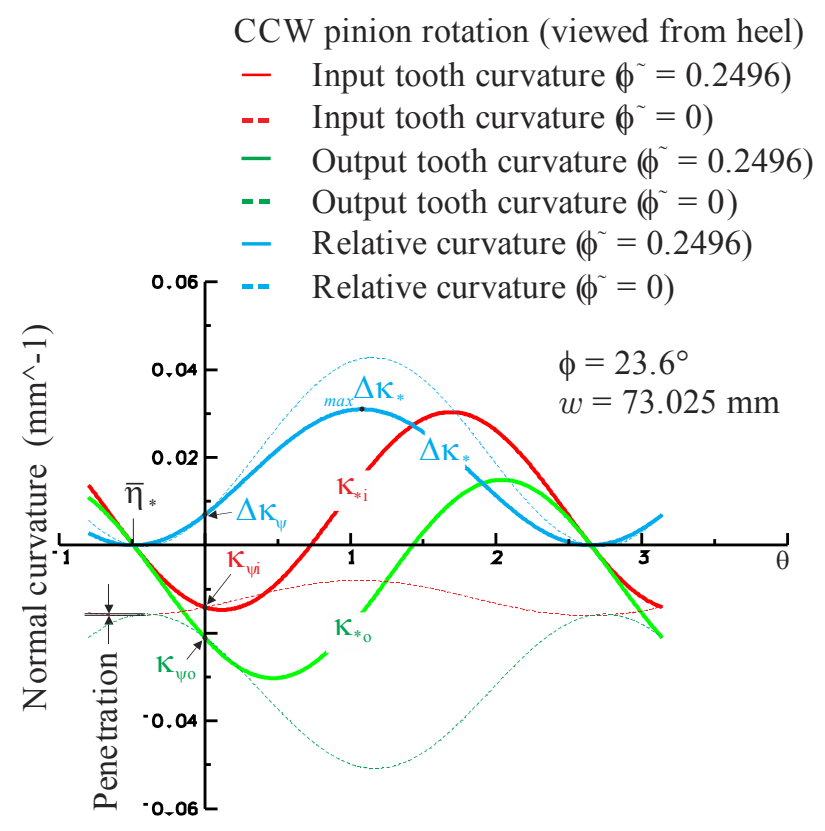

Angle relative to spiral tangent (radians)

Fig. 14. Normal curvature for input and output gear elements.

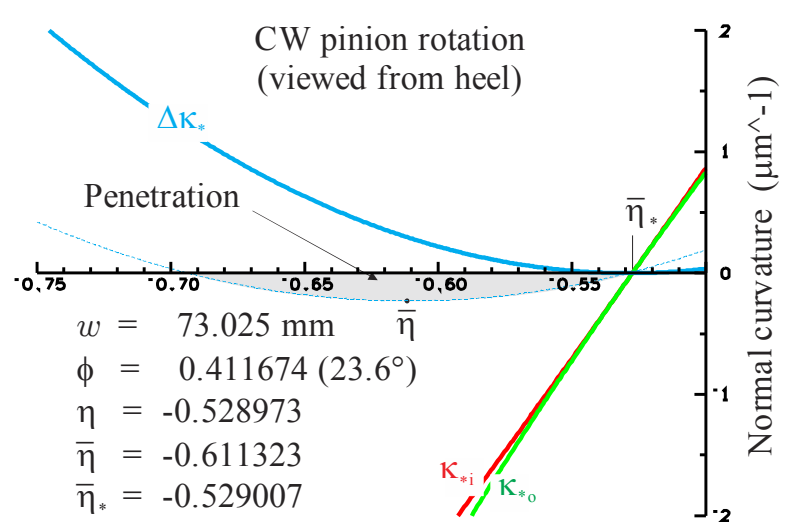

Angle relative to spiral tangent (radians)

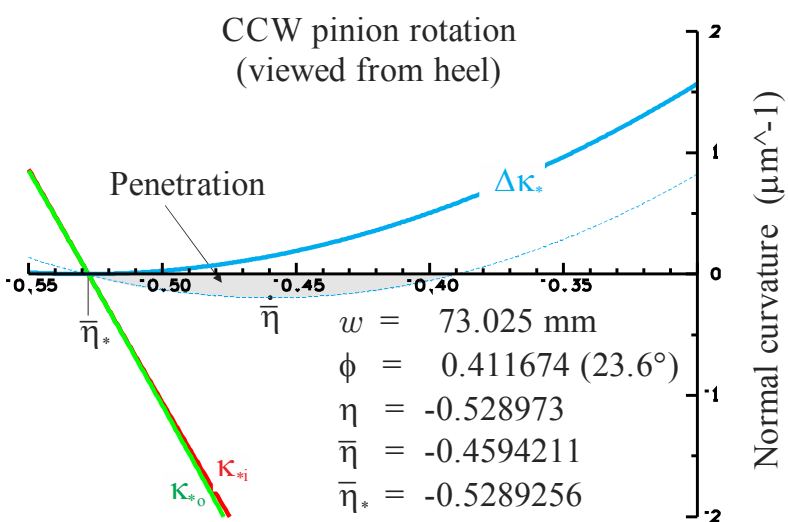

Angle relative to spiral tangent (radians)

Fig. 15. Zoomed-in views of penetration for flanks with constant pressure angle. 


\section{Profile modification}

Tooth contact for gears with cylindrical and conical pitch surfaces can be decomposed into transverse motion and axial motion. Transverse motion considers how the contact moves in a transverse surface or transverse direction while axial motion considers how the contact moves in the axial surface or axial direction. Although profile modification can be any shape, presented will be a quadratic transverse profile modification and quadratic axial profile modification. Modification is centered anywhere along the flank pitch point between toe and heel.

Profile modification is typically used in cylindrical (spur and helical) gears to accommodate non-ideal mesh (deflections and manufacturing tolerances). This same reasoning applies to hypoid gears. Transverse profile modification is illustrated in Fig. 16. Each tooth pitch is divided into five regions as identified in Fig. 16. Regions II and IV are split into a left-side and a right-side resulting in seven separate relations for profile modification. Transverse tooth profile modification for the seven regions are listed below:

- Region I $\left(0 \leq x \leq x_{e a p}\right)$ :

$$
\delta \Delta_{r}=\frac{\delta_{a}}{\mathrm{P}_{d}}
$$

- Region II-left $\left(x_{e a p_{-} l} \leq x \leq \frac{\pi \cos \phi-\mathrm{B}}{2 \mathrm{~N} \cos \phi}\right)$ :

$$
\delta \Delta_{r}=\frac{\delta_{a}}{\mathrm{P}_{d} x_{\text {eap_l }}^{2}}\left(x-\frac{\pi \cos \phi-\mathrm{B}}{2 \mathrm{~N} \cos \phi}\right)^{2}
$$

- Region II-right $\left(\frac{\pi \cos \phi-\mathrm{B}}{2 \mathrm{~N} \cos \phi} \leq x \leq x_{\text {sap } l}\right)$ :

$$
\delta \Delta_{r}=\frac{\delta_{\mathrm{a}}}{\mathrm{P}_{d} x_{\text {sap_l }}^{2}}\left(x-\frac{\pi \cos \phi-\mathrm{B}}{2 \mathrm{~N} \cos \phi}\right)^{2}
$$

- Region III $\left(x_{\text {sap_l }} \leq x \leq x_{\text {sap_r }}\right)$ :

$$
\delta \Delta_{r}=\frac{\delta_{a}}{\mathrm{P}_{d}}
$$

- Region IV-left $\left(x_{\text {sap_r } r} \leq x \leq \frac{\pi \cos \phi+\mathrm{B}}{2 \mathrm{~N} \cos \phi}\right)$ :

$$
\delta \Delta_{r}=\frac{\delta_{a}}{\mathrm{P}_{d} x_{\text {sap_r }}^{2}}\left(x-\frac{\pi \cos \phi+\mathrm{B}}{2 \mathrm{~N} \cos \phi}\right)^{2}
$$

- Region IV-right $\left(\frac{\pi \cos \phi+\mathrm{B}}{2 \mathrm{~N} \cos \phi} \leq x \leq x_{\text {eap_r } r}\right)$ :

$$
\delta \Delta_{r}=\frac{\delta_{a}}{\mathrm{P}_{d} x_{\text {eap } \_}^{2}}\left(x-\frac{\pi \cos \phi+\mathrm{B}}{2 \mathrm{~N} \cos \phi}\right)^{2}
$$

- Region $\mathrm{V}\left(x_{\text {eap_r } r} \leq x \leq \frac{2 \pi}{\mathrm{N}}\right)$ :

$$
\delta \Delta_{r}=\frac{\delta_{a}}{\mathrm{P}_{d}} .
$$

Gear teeth with transverse profile modification are calculated by replacing $\Delta_{r}$ with $\Delta_{t o t}$ in Eq. (5) where

$$
\Delta_{t o t}=\Delta_{r}-\delta \Delta_{r}
$$

Shown in Fig. 17 are the original tooth surface determined using Eq. (5) and the modified surface using (33).

Axial profile modification is obtained by varying backlash. Nominal backlash is specified in terms of a dimensionless backlash constant $B$ [12]. Although many suitable functions exists to define lead crown, polynomials are used to quantify axial lead crown. Shown in Fig. 18 are quadratic lead crown where the nominal contact is centered at the mid-face and where the nominal contact is shifted towards the heel. Also shown in Fig. 18 is quartic lead crown. In all three cases the axial modification is defined at the heel in terms of the nominal diametral pitch. The reference axial position $w_{0}$ is defined accordingly: 


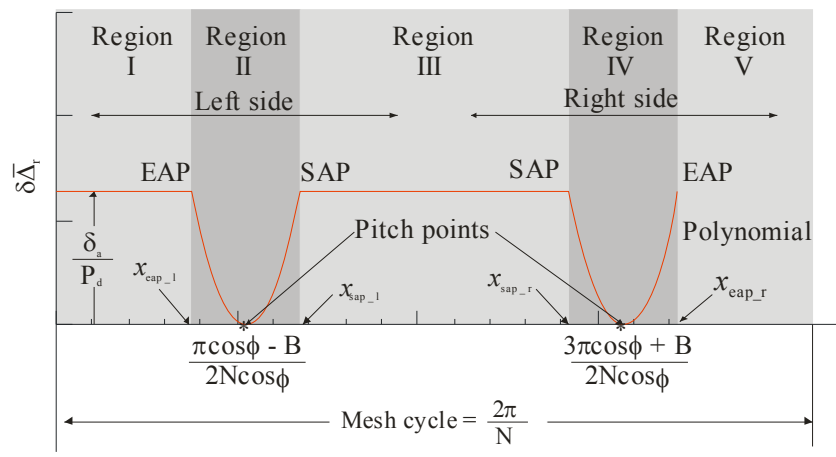

Fig. 16. Transverse profile modification.

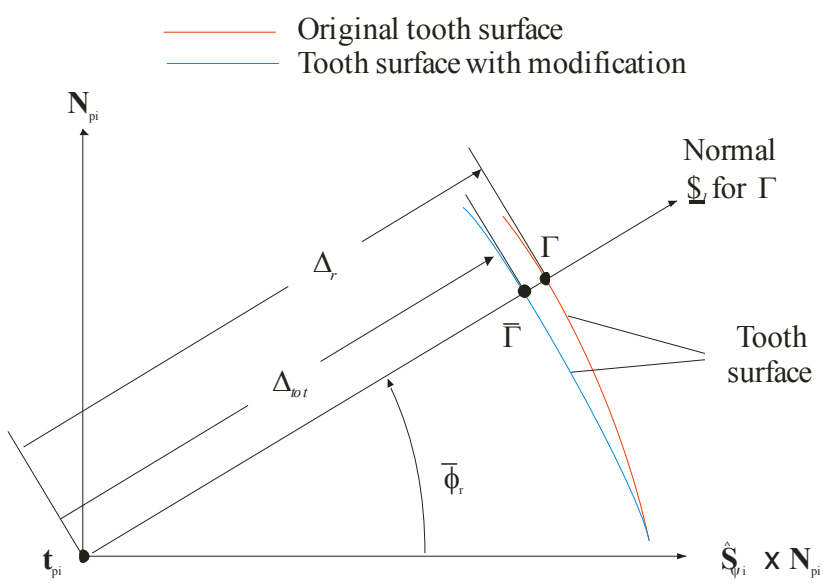

Fig. 17. Normal pressure angle (polar plane).

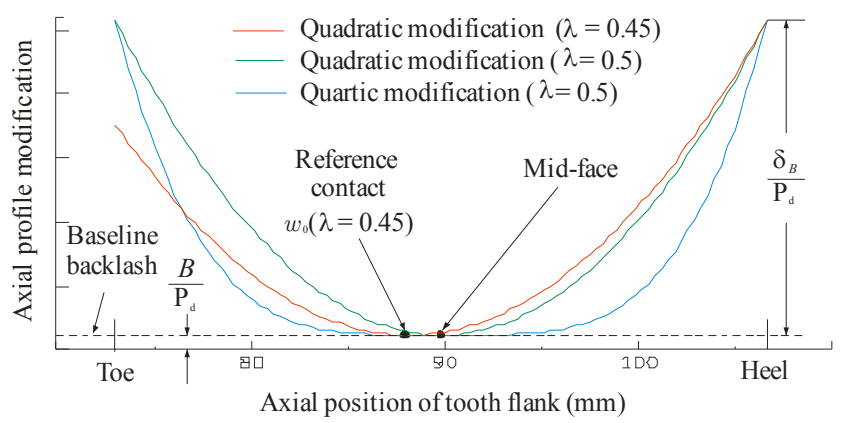

Fig. 18. Axial profile specification using backlash.

$$
w_{0}=(1-\lambda) w_{T o e}+\lambda w_{H e e l}
$$

where $0.0 \leq \lambda \leq 1.0$. A quadratic polynomial $p(w)$ to specify modification in backlash becomes

$$
p(w)=a_{2} w^{2}+a_{1} w+a_{0}
$$

where

$$
\left[\begin{array}{l}
a_{2} \\
a_{1} \\
a_{0}
\end{array}\right]=\left[\begin{array}{ccc}
w_{0}^{2} & w_{0} & 1 \\
2 w_{0} & 1 & 0 \\
w_{\text {Heel }}^{2} & w_{\text {Heel }} & 1
\end{array}\right]^{-1}\left[\begin{array}{c}
p\left(w_{0}\right)=0 \\
\frac{d p}{d w}\left(w_{0}\right)=0 \\
p\left(w_{\text {Heel }}\right)=\frac{\delta_{\mathrm{B}}}{\mathrm{P}_{\mathrm{d}}}
\end{array}\right] .
$$

Backlash $\Delta_{\mathrm{B}}$ for each coordinate curve becomes

$$
\Delta_{\mathrm{B}}=\frac{\mathrm{B}}{\mathrm{P}_{\mathrm{d}}}+p\left(w_{0}\right) \text {. }
$$

Fig. 13 illustrates the UEO topography for the gear flanks without any profile modification. Profile modification can be applied to only the input gear element, only the output gear element, or distributed between the gear elements. In the 
presented example, axial and transverse profile modifications are equally applied to both gear elements. Fig. 19a and Fig. $19 \mathrm{~b}$ depict UEO topography with second order transverse tooth profile modification whereas Fig. 20a and Fig. 20b depict UEO topography for flank geometry with fourth order axial modification. Fig. 21 shows UEO for the gear flanks with combined quadratic transverse profile modification and quartic axial profile modifications. Presented in Fig. 22a and Fig. 22b are UTE for the hypoid gear set with unmodified flanks whereas Fig. 23a and Fig. 23b are UTE for the modified flanks.

Unloaded TE results are based on rigid body analysis of the gear mesh. Such analysis provides a fundamental step in establishing flank geometry. In order to account for gear manufacturing and assembly tolerances, deflections encountered in operation, variations in lubricant film thickness, thermal expansion of the gear elements along with other sources of non-conjugate mesh, loaded TE is presented next to provide a more accurate assessment on the presented methodology for defining fully conjugate hypoid tooth flanks.

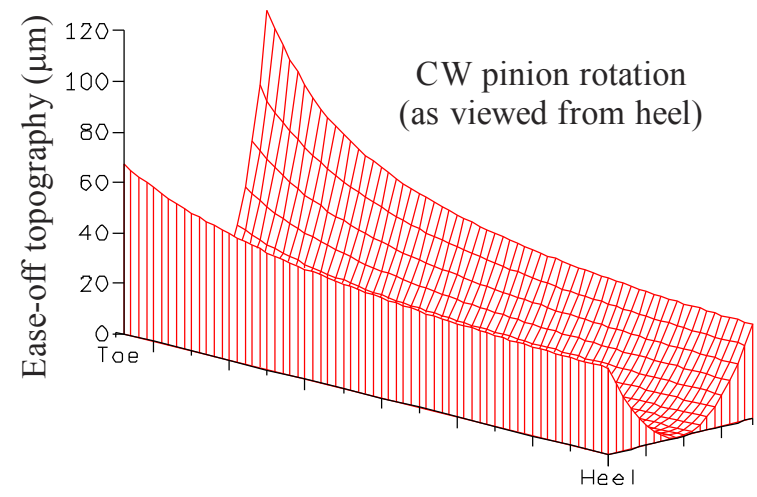

(a) Transverse profile modification (left side)

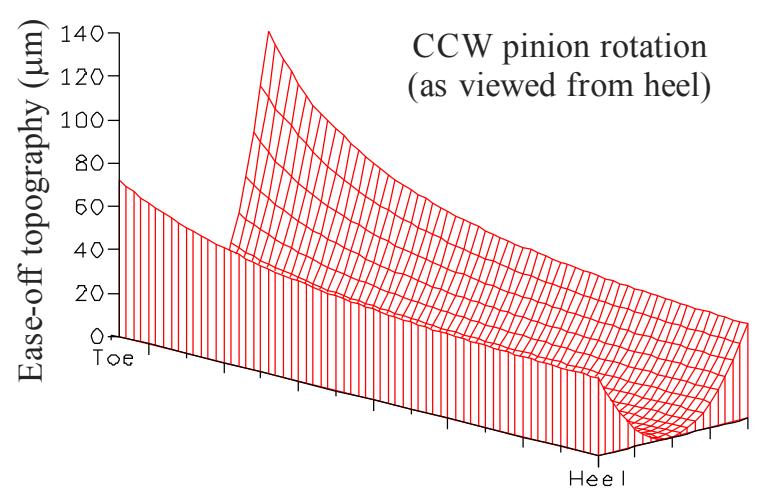

(b) Transverse profile modification (right side)

Fig. 19. Second order transverse Ease-off topography for gear set using data in Table 2.

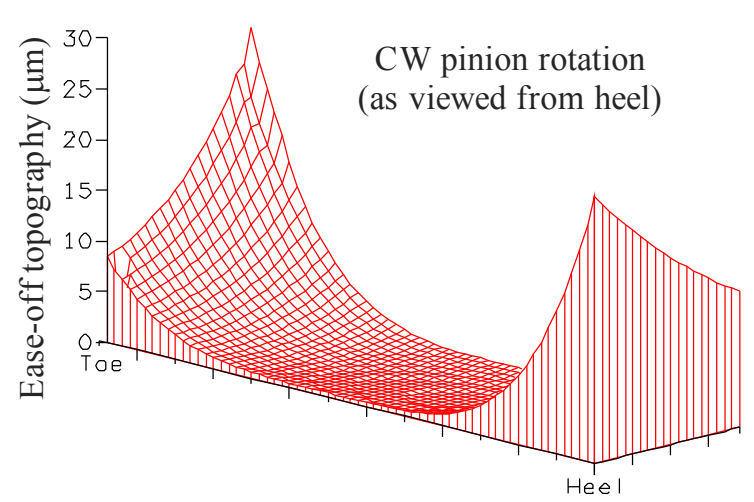

(a) Axial profile modification (left side)

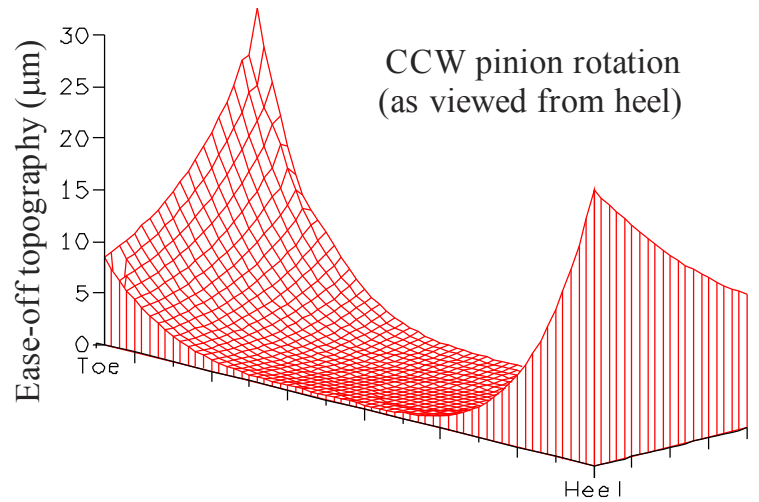

(b) Axial profile modification (right side)

Fig. 20. Fourth order axial Ease-off topography for gear set using data in Table 2.

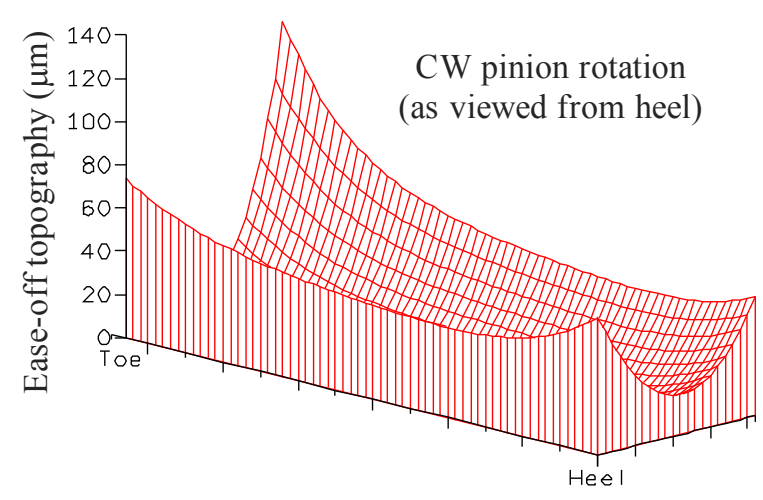

(a) Full profile modification (left side)

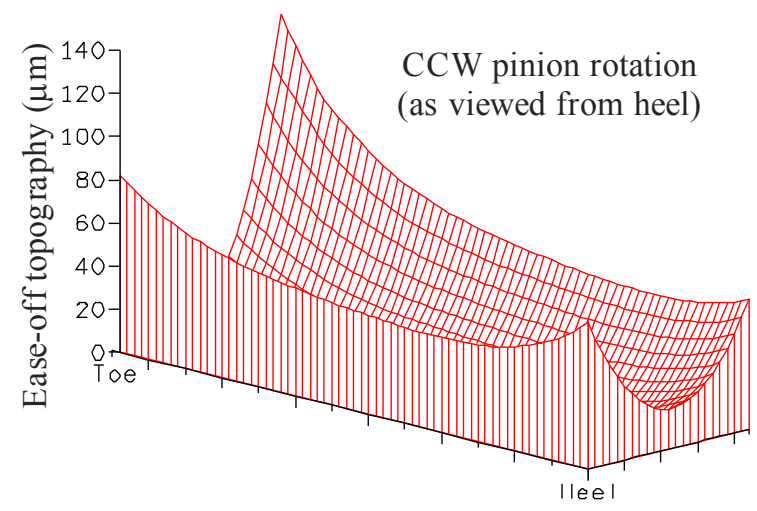

(b) Full profile modification (right side)

Fig. 21. Full Ease-off topography for gear set using data in Table 2. 

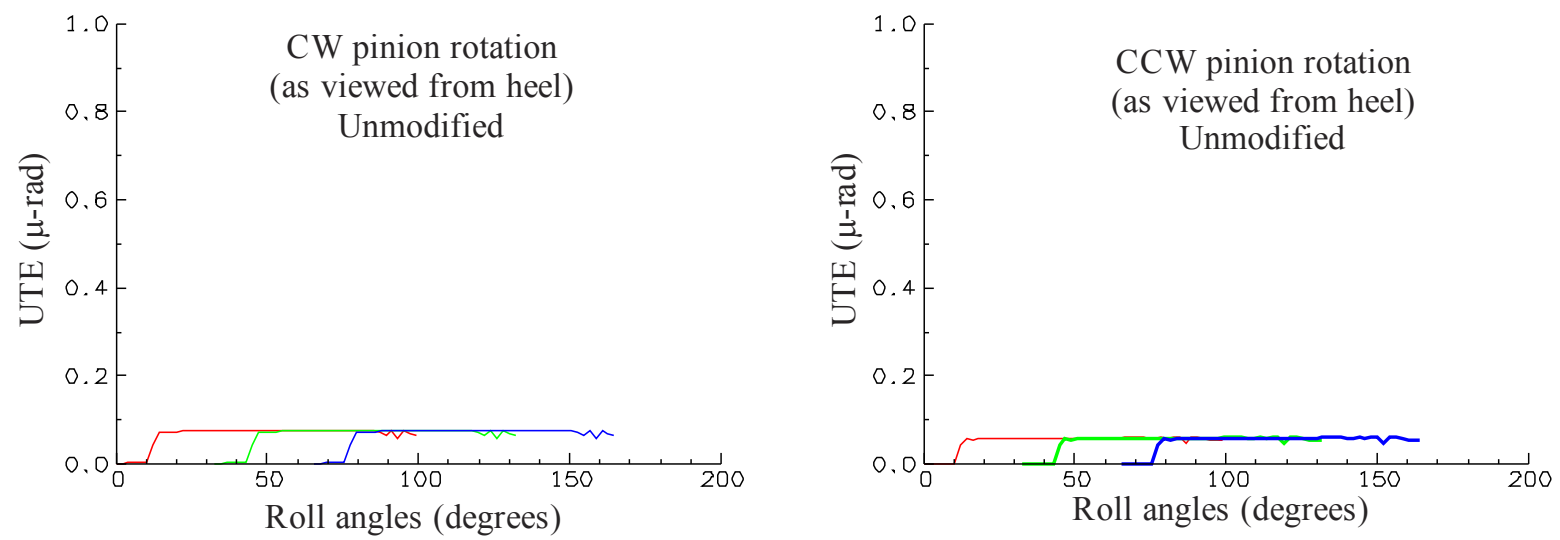

Fig. 22. UTE for unmodified hypoid gear flanks using data of Table 2.

$\mathrm{CW}$ pinion rotation (as viewed from heel)
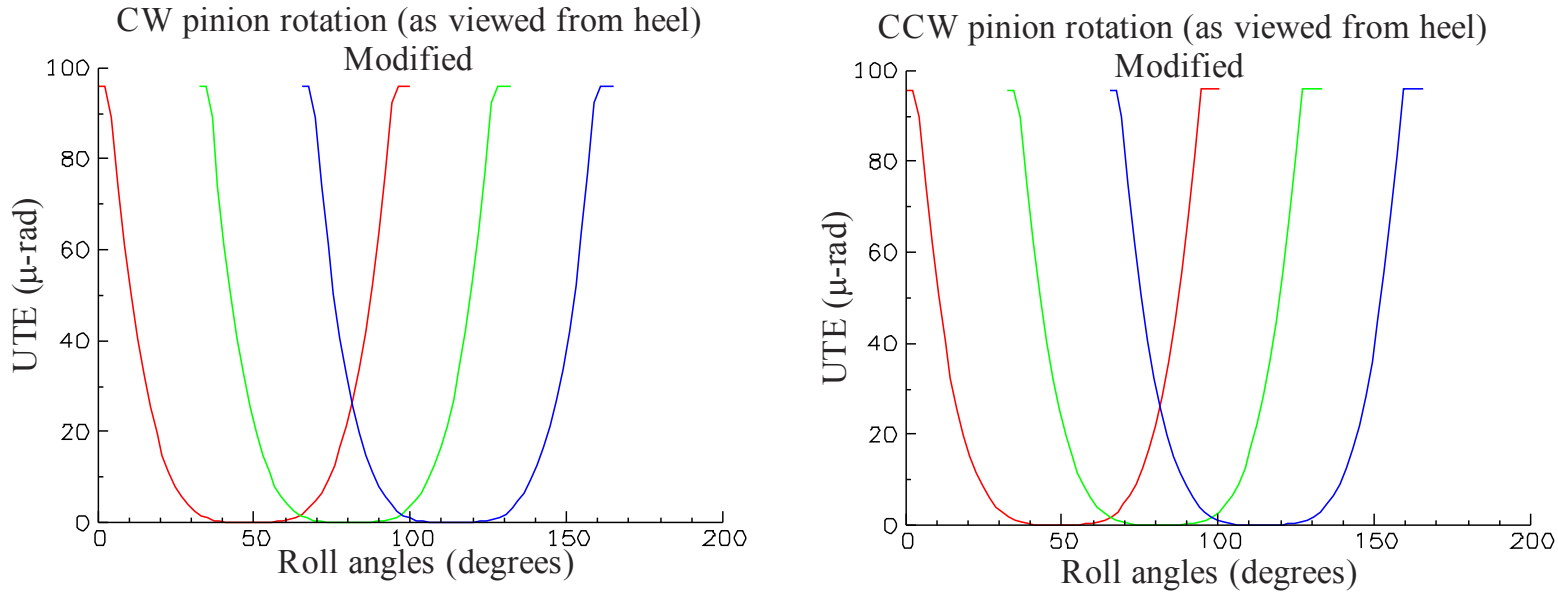

Fig. 23. UTE for modified hypoid gear flanks using data of Table 2. 


\section{Tooth Contact Analysis}

The vibrational behavior of two mating gears can be analyzed by computerized and numerical procedures of tooth contact analysis. The performance of the motion transmission is strictly related to the gear tooth profiles and to the phenomena that occur when two mating gears come into contact. Nonlinear problems arise due to the variability of contact mechanics and conditions. Analytical solutions are not available for complex problems of contact mechanics and consequently numerical simulations represent a helpful instrument for the topic. Accordingly, over the last decades of the twentieth century, Tooth Contact Analysis (TCA) has been generally accompanied by Finite Element Analysis (FEA) [10] or analytical multibody methods [18].

Concerning this work, nonlinear FE-based contact simulations are performed through the commercial software package NX Nastran (SOL 601) [19], in order to evaluate the contact performance of the gear pair in terms of Static Transmission Error (STE) and overall contact pattern.

Although such a FE-based methodology for TCA is relatively expensive in terms of computational time, it does guarantee an accurate computation of TE. A quasi Unloaded Tooth Contact Analysis (UTCA) is computed by applying a low torque, in order to consider the kinematic characteristics of the meshing gear elements. On the other side, results of a Loaded Tooth Contact Analysis (LTCA) are presented to provide a more realistic description of the gear pair contact behavior, including the tooth bending deflections that modify the overall contact conditions and thus the STE trend.

The results of the described analysis show that the analyzed tooth profiles are close to conjugate.

\subsection{FE model creation}

The FE model of the hypoid gear pair is created using a dedicated preprocessing software tool, which is an in-house code that allows to obtain highly detailed and customized FE models starting from a cloud-of-points based representation of the gear flanks and from the gear blank data. The discretization of the gear geometry (blank and teeth) is obtained as a set of six-sided solid elements (HEXA8). Since static nonlinear simulations are expected to require significant computational time, the FE model of each gear pair element is characterized by a combination of three FE mesh sections, each with its own mesh density:

- A finer part is ascribed to the teeth that most likely enter in contact as the load increases. A high number of elements is needed to accurately detect contact between the mating flanks and describe the deformation gradients in the contact zone.

- A very coarsely meshed section is generated for most of the gear that will not be in contact during the simulations. It is used only to correctly approximate the overall blank stiffness without introducing additional and unrequested degrees of freedom (DOFs) to the model.

- A third part is made up of two small intermediate sections (one on each side) that connect the fine and the coarse mesh parts. Its main role is avoiding FE mesh discontinuities, which could provide misleading results or convergence instability.

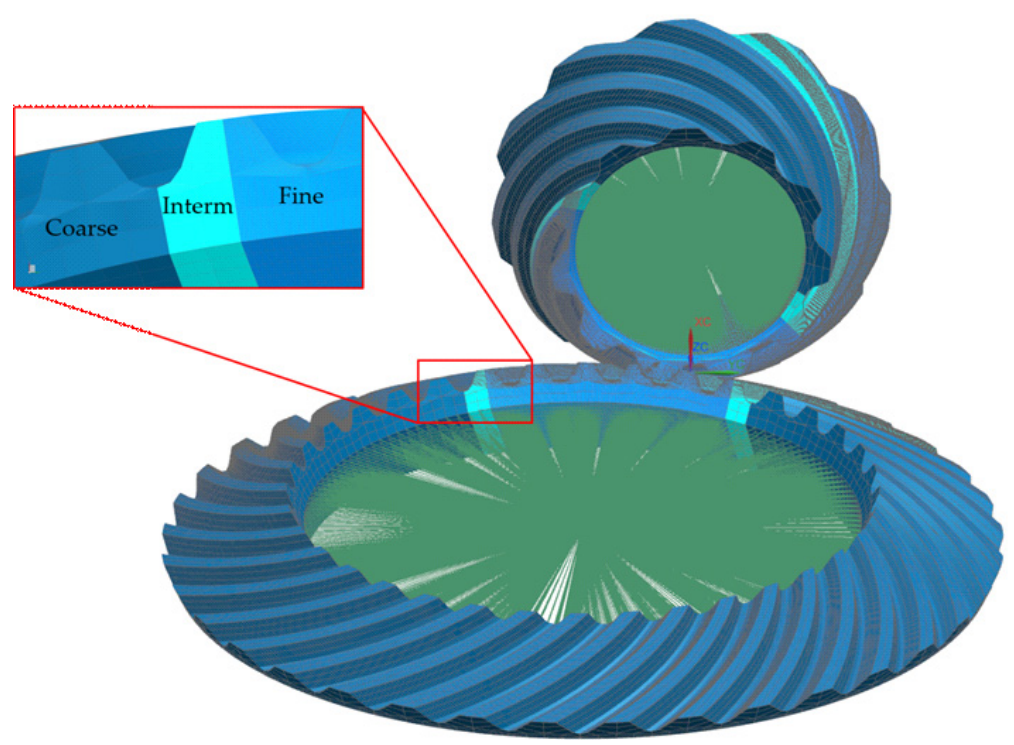

Fig. 24. The finite element model of the analyzed hypoid gear pair. 
As depicted in Fig. 24, 2D Rigid Body Elements (RBE2) are adopted for the FE models of both gear and pinion to constrain the nodes that make up the bore. They act as multiple point constraints (MPCs) in order to force the same boundary conditions to all the nodes that lie on the inner cones of the gear pair elements. During the simulation, the driven gear's RBE2 center node is then kept fully constrained, while the RBE2 center node of the pinion is constrained in such a way that the pinion is allowed to only rotate about its rotational axis. The external torque is applied as acting on the pinion's rotational axis.

In the presented work, TCA has been carried out for five different load cases: $1 \mathrm{Nm}, 50 \mathrm{Nm}, 100 \mathrm{Nm}, 250 \mathrm{Nm}$ and $500 \mathrm{Nm}$. The $1 \mathrm{Nm}$ load case has been considered as the "unloaded case".

A standard steel has been assigned as material of the elements of both pinion and gear, with E $=200$ GPa (Young's Modulus) and $\nu=0.33$ (Poisson's ratio).

\subsection{Contact detection}

The adopted contact detection strategy takes place from a FE discretization of the contact interfaces, such as the tooth active flanks. In this work, it has been used the so-called node-to-surface approach [18], which relies on the definition of two kinds of contact surfaces: the slave and the master surfaces. According to the considered method, the nodes of the slave surface cannot penetrate the segments of the master surface. The selection of these regions is relevant and results from several considerations about the mesh size, the surface curvatures and the material. Here, the pinion has been chosen to be the slave, while the driven gear has been selected to be the master.

The node-to-surface method provides finding the point $\boldsymbol{x}^{(M)}(\xi, \eta)$ on the master surface $\Sigma^{(M)}$ that makes minimum the distance between a given node $\boldsymbol{x}^{(S)}$ of the slave surface $\Sigma^{(S)}$ and the master surface $\Sigma^{(M)}$ [18]. This distance is called gap $(g)$ and it can be expressed as:

$$
g=\left[\boldsymbol{x}^{(S)}-\boldsymbol{x}^{(M)}(\xi, \eta)\right] \cdot \boldsymbol{n}^{(M)},
$$

where the gap $g$ is computed as the distance between the position vectors of the slave point $\boldsymbol{x}^{(S)}$ and the master point $\boldsymbol{x}^{(M)}$, projected onto surface normal $\boldsymbol{n}^{(M)}$ that is assessed at the contact point on the master surface. If no friction is considered during the contact, the Hertz-Signorini-Moreau (HSM) condition, presented in Eq. (38), can be used as nopenetration condition, in order to impose contact along the normal direction [20].

$$
g_{N} \geq 0, \quad \lambda \leq 0, \quad \lambda \cdot g_{N}=0,
$$

While a gap is detected, there cannot be a contact force. The latter is expressed in the form of the Lagrange multiplier $\lambda$. Vice versa, if a contact force is identified, the gap must be null. In the considered numerical solution [18], the HSM-condition is replaced by the constraint function presented in Eq. (39), where $\epsilon_{N}$ is a small parameter, defined by the user.

$$
w\left(g_{N}, \lambda\right)=\frac{g_{N}+\lambda}{2}-\sqrt{\left(\frac{g_{N}+\lambda}{2}\right)^{2}-\epsilon_{N}},
$$

It is possible to assign a finite compliance to the contact surface. Thus, a user-specified amount of interpenetration between the contacting surfaces is allowed, in order to simulate the contact between soft or compliance surfaces. The amount of penetration $\delta_{p}$ is defined as:

$$
\delta_{p}=\epsilon_{p} p_{N}=\epsilon_{p} \frac{\lambda}{A}
$$

where $\epsilon_{p}$ is the contact surface compliance parameter and $p_{N}$ the normal surface contact pressure. Fig. 25 shows how the constraint function $w\left(g_{N}, \lambda\right)=0$ is altered by introducing the contact compliance $\epsilon_{p}$. The latter allows to emulate the presence of an unidirectional spring with stiffness constant $A / \epsilon_{p}$, where $A$ is the contact surface and $\lambda$ the contact force that is applied on the contact interface.

Earlier obtained results indicated a maximum contact pressure of about $3000 \mathrm{MPa}$ at the maximum load of $500 \mathrm{Nm}$. Such a high value of the maximum contact pressure and discontinuous contact patterns induced to introduce a compliance to the contact surfaces.

Taking into account the size of the gear pair, $\delta_{p}=1.5 \mu \mathrm{m}$ of interpenetration has been allowed between both surfaces, yielding a contact compliance value equal to:

$$
\epsilon_{p}=5 \times 10^{-10} \frac{\mathrm{mm}^{3}}{\mathrm{mN}} .
$$




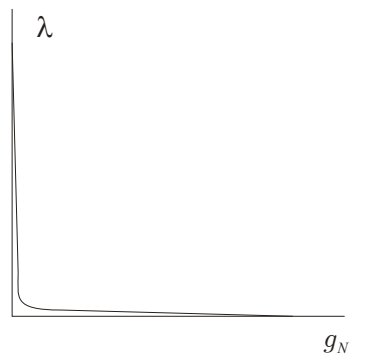

(a)

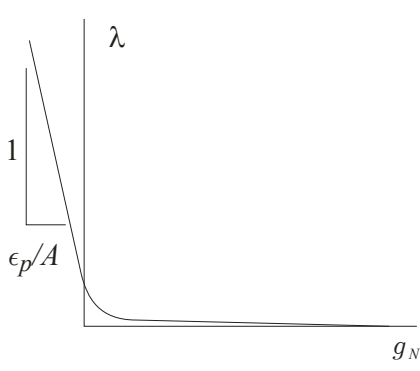

(b)

Fig. 25. (a) Constraint function without contact compliance; (b) constraint function with contact compliance.

\subsection{Kinematic configuration}

Each geared body has to be analyzed in its own kinematic configuration in order to obtain high power transmission performance. Conjugate motion consists of perfect motion transfer that can be maintained for every considered roll angle during a whole meshing cycle. The latter is defined as

$$
\phi_{m c}=\frac{2 \pi}{z},
$$

where $\phi_{m c}$ is expressed in radians and $z$ is the number of teeth of the considered gear. In this work, the results refer to the pinion's meshing cycle that is equal to about 0.571 rads. However, gears have to transmit a certain load during the engagement. Since they are not characterized by infinite stiffness, their profile elastically deforms, giving rise to an unsteady component in the relative angular motion of the mating gears. This component is due to the periodic variation in the stiffness of the gear mesh that can be mainly ascribed to the fluctuation of the contact ratio. The latter is the instantaneous number of teeth in contact during the rotation. Perfect motion cannot be transmitted due to the varying mesh stiffness. The condition of non-conjugacy is one of the main sources of dynamic excitation that a gear design tries to minimize at operating conditions.

The STE is an index of the excitation that occurs on the geared systems and of their performances. It considers the difference in rotation between the actual position of the output gear and the position that the gear would occupy in case of perfect drive.

$$
S T E=\phi_{2}-\frac{z_{1}}{z_{2}} \phi_{1}=\phi_{2}-\tau \phi_{1}
$$

where $\phi_{1}$ and $\phi_{2}$ represent respectively the rotational angles of the pinion and the driven gear around the correspondent axis of rotation; $z_{1}$ and $z_{2}$ are the numbers of teeth and $\tau$ the transmission ratio. In presence of pure kinematic motion, the STE should be ideally equal to zero.

About the starting configuration of the static simulations, the gear pair is oriented in such a way that the mating flanks are closed to contact. Table 3 gives an overview of the initial rotational angles for the FE models of the presented gear pair elements.

Table 3. Starting configuration of the gear pair elements.

\begin{tabular}{cc}
\hline Gear element & $\begin{array}{c}\text { Initial rotational angle } \\
\text { [rad] }\end{array}$ \\
\hline Pinion & 2.82292931 \\
Gear & 0.05443122 \\
\hline
\end{tabular}

\subsection{STE results}

Using the previously described approach, it has been possible to compute the tooth contact analysis resulting in the STE curves depicted in Fig. 26. The STE curves refer to both the unmodified and modified models and they have been represented making null their mean value, in order to appreciate the increase of the peak-to-peak values for higher loads. The results show an almost flat behavior of the quasi Unloaded Transmission Error (UTE), which attests the conjugate nature of the mating flanks. The Loaded Transmission Error (LTE) curves are characterized by increasing peak-to-peak values, because of tooth bending contribution, due to the applied torques. For each load, the highest value of STE is obtained at the middle of the mesh cycle. In this configuration three tooth pairs carry the load while other tooth pairs are disengaged. At the beginning and at the end of mesh the cycle, a decrease in the STE is noticed. This is ascribed to an 
increase in the overall mesh stiffness as the result of improved load sharing between the flanks of four tooth pairs in contact.

As it is remarked in Table 4, a slight increase in peak-to-peak TE values is obtained for the modified model. The modification applied on the active flank consists in removing material as described in Section 6 . A variation of the contact conditions occurs and different STE values and contact pressure distribution are expected.

The applied profile modification produces a beneficial effect on the overall contact pattern and resulting contact pressures. Fig. 27 shows a comparison of the contact pressures for both the unmodified and modified models, considering different applied loads. It can be noticed that edge contact occurs for the unmodified model, while a better pressure distribution is obtained in the modified one. The results demonstrated that the mating flanks have fully conjugate profiles, since the quasi unloaded transmission error curve is flat. The applied modification allowed to achieve a better contact pressure distribution, avoiding the occurrence of edge contact.

Only the case of the drive side for STE and contact pressures are presented. For the automotive hypoid gear set presented, the drive side corresponds to a CW rotation of the input gear element (viz., the pinion) as viewed from the heel. Such contact involves the concave side of the input tooth flank and the convex side of the output gear flank. It is interesting to note that maximum relative curvature $\max _{*} \Delta \kappa_{*}$ increases for $\mathrm{CW}$ pinion rotation when removing penetration whereas $\max _{*} \Delta \kappa_{*}$ decreases for CCW pinion rotation (see Fig. 14).

Table 4. Peak-to-peak STE values for the unmodified and modified models.

\begin{tabular}{|c|c|c|c|c|c|}
\hline 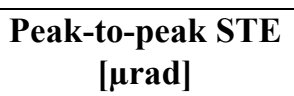 & $1 \mathrm{Nm}$ & $50 \mathrm{Nm}$ & $100 \mathrm{Nm}$ & $250 \mathrm{Nm}$ & $500 \mathrm{Nm}$ \\
\hline Unmodified & 4.91 & 31.56 & 51.75 & 98.61 & 151.05 \\
\hline Modified & 20.48 & 36.58 & 63.66 & 116.07 & 171.20 \\
\hline
\end{tabular}

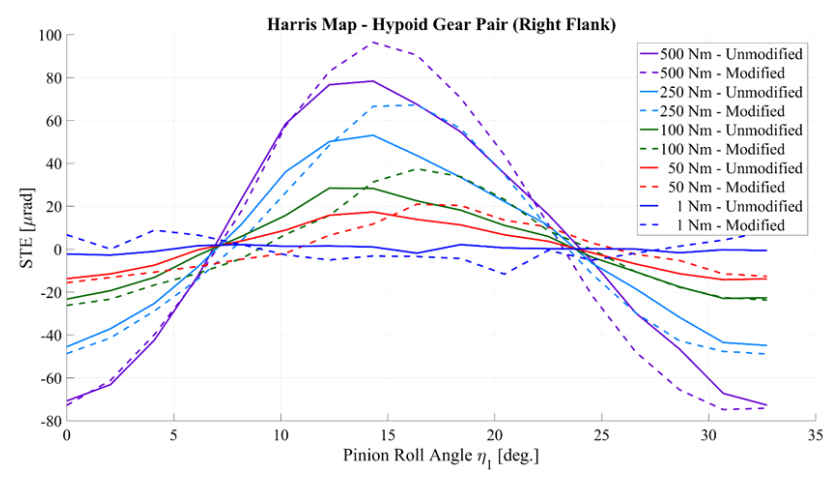

Fig. 26. STE curves for different torques applied on both the unmodified and modified gear pairs.

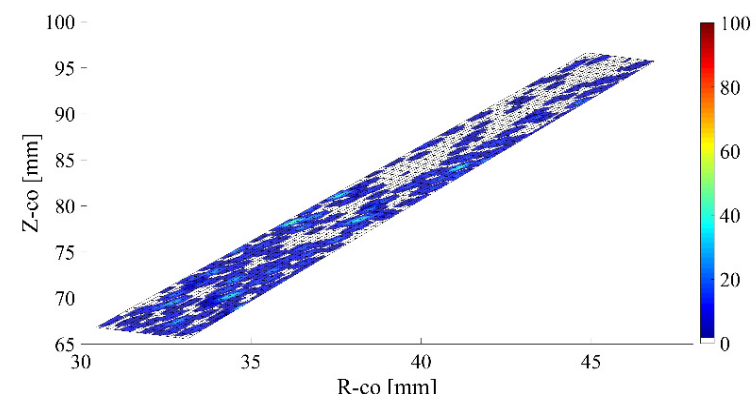

(a) Overall contact pressures - $1 \mathrm{Nm}$ - Unmodified

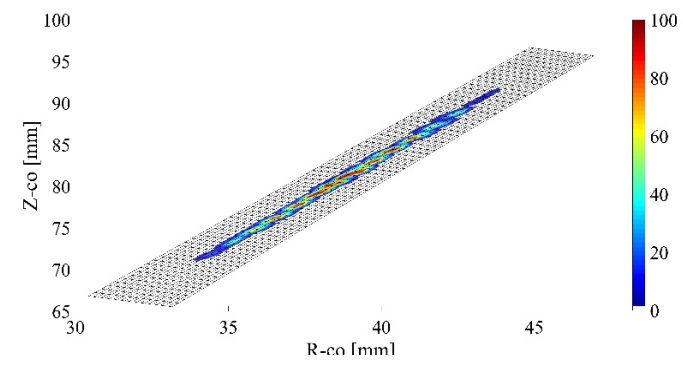

(b) Overall contact pressures - $1 \mathrm{Nm}$ - Modified 

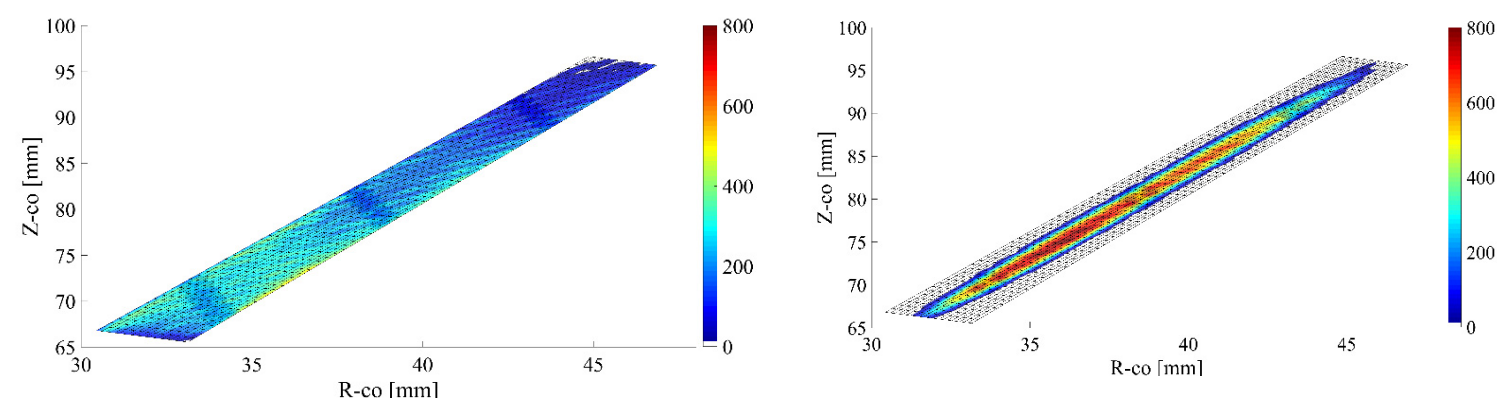

(c) Overall contact pressures - $50 \mathrm{Nm}$ - Unmodified (d) Overall contact pressures - $50 \mathrm{Nm}$ - Modified
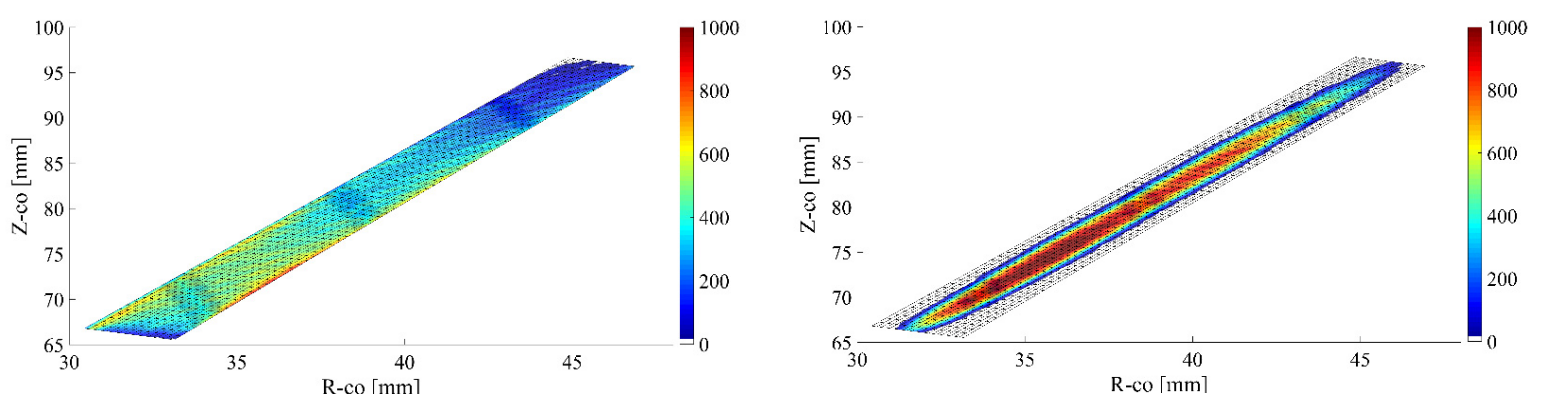

(e) Overall contact pressures - $100 \mathrm{Nm}$ - Unmodified (f) Overall contact pressures - $100 \mathrm{Nm}$ - Modified
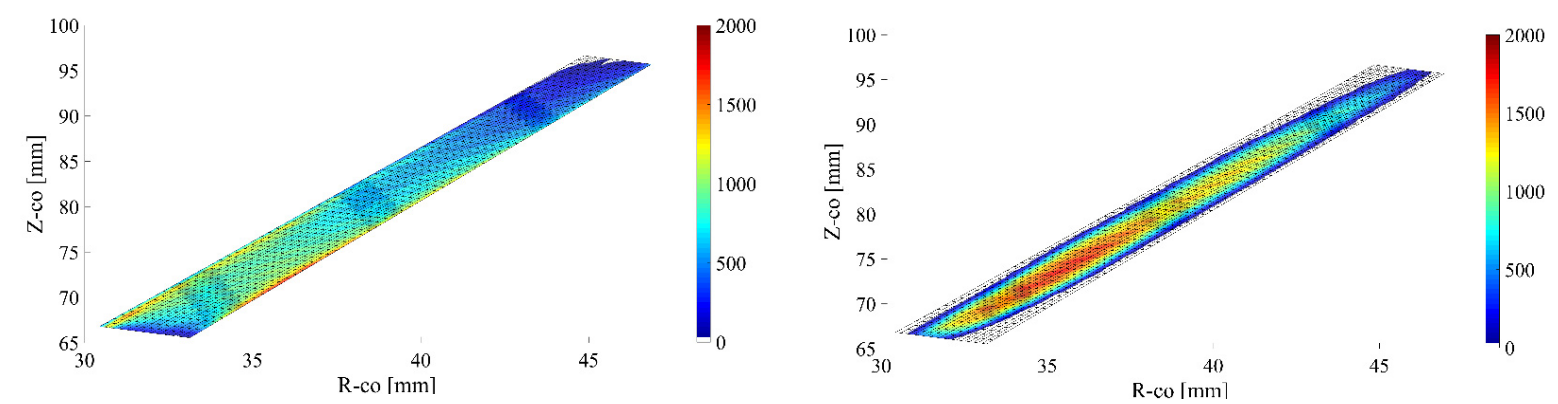

(g) Overall contact pressures - $250 \mathrm{Nm}$ - Unmodified (h) Overall contact pressures - $250 \mathrm{Nm}$ - Modified
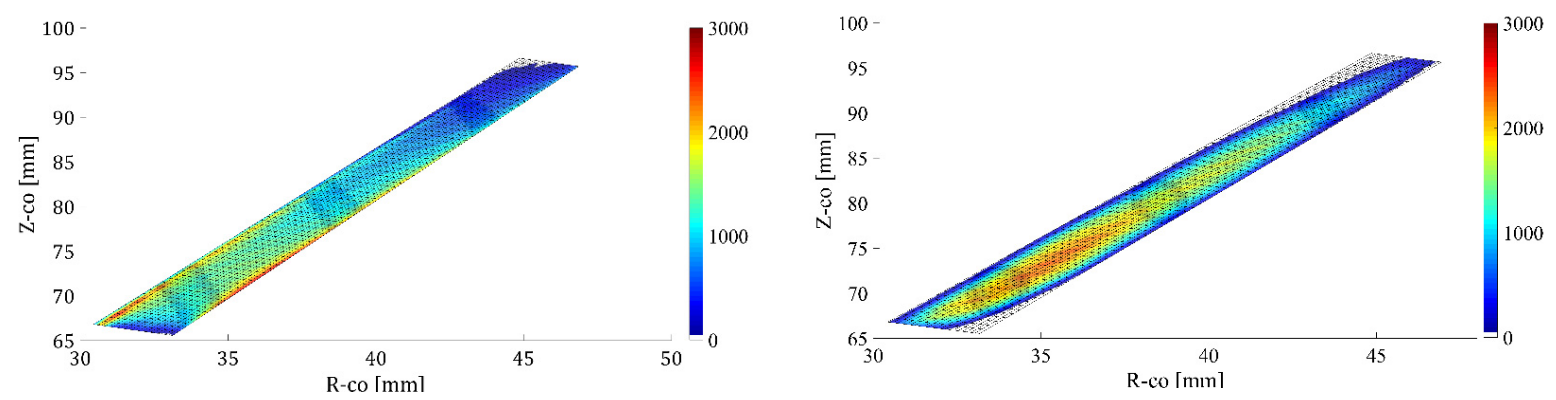

(i) Overall contact pressures - $500 \mathrm{Nm}$ - Unmodified (j) Overall contact pressures - $500 \mathrm{Nm}$ - Modified

Fig. 27. FE-based contact results for $1 \mathrm{Nm}-50 \mathrm{Nm}-100 \mathrm{Nm}-250 \mathrm{Nm}-500 \mathrm{Nm}$.

Comparison of contact pattern and contact pressure for both unmodified and modified models. 


\section{Conclusions}

Presented is the first known procedure to directly specify fully conjugate tooth flanks for hypoid gear elements. This procedure uses a basic involute rack "rolling" in the null plane to specify flank coordinates. Central to this process is an axial variation in the normal pressure angle that satisfies first order reciprocity between the flank contact normal and the instantaneous twist defined by the gear rotation axes and gear ratio. The procedure enables the determination of gear teeth in a fashion akin to the specification of cylindrical spur and helical gears. This methodology extends Deproximating Tredgold's Approximation and enables fully conjugate gear teeth to be specified for hypoid gears using a planar involute rack profile. Zero unloaded Ease-off topography for the gear flanks was demonstrated for a rear axle automotive gear set. The contact pattern evenly spanned the tooth flank using the presented methodology. Generalized axial and transverse profile modifications were established for a reference contact point on the tooth flank. Unloaded TE was presented for flanks without and with micro-modifications. Subsequently, an in-depth simulation of the mesh with loaded TE was presented as a virtual experiment to provide a more accurate representation of TE together with a more comprehensive assessment of the presented flank geometry. STE curves were generated for a family of input torques for both the unmodified and modified flanks. Additionally, the overall contact pattern was presented for these loading scenarios. In conclusion, fully conjugate flanks were generated together with flanks incorporating polynomial transverse and axial modifications.

\section{Acknowledgements}

This research did not receive any specific grant from funding agencies in the public, commercial, or not-for-profit sectors.

\section{References}

[1] Dooner, D.B., Vivet, M., and Mundo, D., "Deproximating Tredgold's approximation," Mechanism and Machine Theory, 102, pp. 36-54, 2016.

[2] Dooner, D.B., "On the Third Law of Gearing: a study on hypoid gear tooth contact," Mechanism and Machine Theory, 134, pp. 224-248, 2019.

[3] Stadtfeld, H.J., Handbook of Bevel and Hypoid Gears, Rochester Institute of Technology, Rochester, 1993

[4] Grant, G.B., 1899, A Treatise on Gear Wheels, Grant Gear Works, Boston, Massachusetts, 1899.

[5] Struik, D.J., Lectures on Classical Differential Geometry, Addison-Wesley Publishing Co., Reading Mass.; republished by Dover Publication Inc., Meneola N.Y., 1961, 1988.

[6] Hunt, K.H., Kinematic Geometry of Mechanisms, Clarendon, Oxford, England, 1978.

[7] Bottema, O., and Roth, B., Theoretical Kinematics, North Holland Publishing, Amsterdam, Holland, 1979.

[8] Colbourne, J.R., The Geometry of Involute Gears, Springer-Verlag New York Inc., Woodbine, New Jersey, 1987.

[9] Phillips, J.R., General Spatial Involute Gearing, Springer, Berlin, Germany, 2003.

[10] Litvin, F.L., and Fuentes, A., Gear Geometry and Applied Theory, 2nd ed. Cambridge University Press, London, 2004.

[11] Figliolini G., Stachel H., Angeles J. (2009) The Computational Fundamentals of Spatial Cycloidal Gearing. In: Kecskeméthy A., Müller A. (eds) Computational Kinematics. Springer, Berlin, Heidelberg.

[12] Dooner, D.B., Kinematic Geometry of Gearing, 2nd edition, Wiley, London, 2012.

[13] Dooner, D.B., 2013, "Hobbing of Bevel and Hypoid Gears," ASME Power Transmission and Gearing 
Conference, Aug. 4-7, Portland, Oregon.

[14] Figliolini, G., Stachel, H., Angeles, J., "Kinematic properties of planar and spherical logarithmic spirals: Applications to the synthesis of involute tooth profiles," Mechanism and Machine Theory, 136, pp. 1426, 2019.

[15] Dooner, D.B., and Winfough, W.R., Testing of Alternative Spiral Bevel and Hypoid Gear Theory," VDI Conference, Oct. 7-9, Munich, Germany, 2013.

[16] Dooner, D.B., Vivet, M., 2018, "Unloaded Tooth Contact Analysis of Hypoid Gears for Increased Power Density," International Gear Conference, Aug. 27-29, Lyon, France.

[17] Figliolini, G., Stachel, H., Angeles, J., "A spatial version of octoidal gears via the generalized Camus theorem," ASME J. of Mechanisms and Robotics, 8 (2), 2015.

[18] Vivet, M., Mundo, D., Tamarozzi, T., Desmet, W., “An analytical model for accurate and numerically efficient tooth contact analysis under load, applied to face-milled spiral bevel gears," Mechanism and Machine Theory, 130, pp. 137-156, 2018.

[19] Siemens Product Lifecycle Management Software Inc., NX Nastran 10. Advanced Nonlinear Solution - Theory and Modeling Guide, 2014.

[20] Wriggers, P., Computational contact mechanics, John Wiley and Sons, 2002.

\section{Nomenclature}

B Dimensionless backlash constant

$\mathbf{C}_{l} \quad$ Direction of tooth contact normal

$\mathbf{C}_{p} \quad$ Direction of pitch surface generator

CAD Computer Aided Design

CCW Counter-clockwise

CNC Computer Numerical Controlled

CW Clockwise

E Shaft offset (Hypoid offset)

EAP End of Active Profile

FEA Finite Element Analysis

g Gear ratio

ISA Instantaneous Screw Axis

$\mathrm{L}_{p} \quad$ Instantaneous lead

LTCA Loaded Tooth Contact Analysis

$m \quad$ Tooth module

$\boldsymbol{n} \quad$ Tooth unit normal

$\mathrm{N}_{i} \quad$ Tooth count for input gear

$\mathrm{N}_{o} \quad$ Tooth count for output gear

$\mathbf{N}_{p} \quad$ Pitch surface normal

OEM Original Equipment Manufacturer

$\mathrm{P}_{d} \quad$ Diametral pitch

SAP Start of Active Profile

$\mathbb{S}_{\psi} \quad$ Spiral tangency

$\boldsymbol{t} \quad$ Tooth tangency

$\mathbf{t}_{p} \quad$ Point on pitch surface

$\mathbf{t}_{p}^{\prime} \quad$ Tangency to transverse curve on pitch surface

STE Static Transmission Error

TCA Tooth Contact Analysis

TE Transmission Error

$(u, v, w)$ Cylindroidal coordinates

$u_{p} \quad$ Throat radius of hyperboloidal pitch surface

UEO Unloaded Ease-off topography 
UTCA Unloaded Tooth Contact Analysis

UTE Unloaded Transmission Error

$v_{i} \quad$ Angular position of gear element

$w_{p} \quad$ Axial position along generator

$x_{\text {eap } \_} \quad$ Rack coordinate for EAP (left side)

$x_{\text {sap } \_} \quad$ Rack coordinate for SAP (left side)

$x_{\text {eap_r }} \quad$ Rack coordinate for EAP (right side)

$x_{\text {sap_ } r} \quad$ Rack coordinate for SAP (right side)

$\left(x_{r}, y_{r}\right) \quad$ Cartesian coordinates of planar cutter

$\left(\bar{x}_{r}, \bar{y}_{r}\right) \quad$ Cartesian coordinates of image $\left(x_{r}, y_{r}\right)$

$\underline{\$}_{l} \quad$ Line of action (tooth contact normal)

$\alpha_{p} \quad$ Generator angle of pitch surface

$\gamma_{p} \quad$ Angle between generator and transverse curve

$\Gamma \quad$ Gear tooth coordinates

$\delta_{a} \quad$ Dimensionless transverse profile relief constant

$\delta_{B} \quad$ Dimensionless axial profile relief constant

$\Delta_{r} \quad$ Chordal distance between contact $\left(x_{r}, y_{r}\right)$ and pitch point $\left(\bar{x}_{r}, \bar{y}_{r}\right)$

$\Sigma \quad$ Shaft angle

$\phi \quad$ Nominal pressure angle

$\bar{\phi}_{r} \quad$ Instantaneous pressure angle

$\psi_{p} \quad$ Pitch surface spiral angle

1 Superscript to designate differentiation w.r.t. angular position $v$

- Superscript to designate differentiation w.r.t. axial position $w$ 


$$
>
$$

